BULLETIN (New Series) OF THE

AMERICAN MATHEMATICAL SOCIETY

Volume 38, Number 1, Pages 43-78

S 0273-0979(00)00886-7

Article electronically published on September 26, 2000

\title{
HARMONIC ANALYSIS WITH RESPECT TO HEAT KERNEL MEASURE
}

\author{
BRIAN C. HALL
}

\begin{abstract}
This paper surveys developments over the last decade in harmonic analysis on Lie groups relative to a heat kernel measure. These include analogs of the Hermite expansion, the Segal-Bargmann transform, and the Taylor expansion. Some of the results can be understood from the standpoint of geometric quantization. Others are intimately related to stochastic analysis.
\end{abstract}

\section{Contents}

1. Introduction

2. Three unitary maps for a compact Lie group 47

2.1. Preliminaries

2.2. The Segal-Bargmann transform for $K$

2.3. The Taylor map

2.4. The Hermite expansion

2.5. Creation and annihilation operators

. The $K$-invariant Segal-Bargmann transform 58

3.1. The transform 58

3.2. The connection with geometric quantization 58

3.3. Phase bounds $\quad 62$

3.4. Compact symmetric spaces 63

4. Connections with the theory for infinite-dimensional linear spaces 64

4.1. Embedding the isomorphisms 64

4.2. The Yang-Mills interpretation 69

5. Proof sketches 72

5.1. The Segal-Bargmann transform for $K$

5.2. The Taylor map 73

5.3. The Hermite expansion 74

References

\section{Introduction}

Parts of harmonic analysis on Euclidean spaces can naturally be expressed in terms of a Gaussian measure, that is, a measure of the form $a \exp \left(-b|x|^{2}\right) d x$,

Received by the editors June $7,2000$.

2000 Mathematics Subject Classification. Primary 22E30, 81S30, 53D50, 60H30; Secondary 43A32, 46E20, 58J25. 
where $d x$ is Lebesgue measure and $a$ and $b$ are positive constants. Among these are logarithmic Sobolev inequalities, Hermite expansions, the quantum harmonic oscillator, coherent states, and the Segal-Bargmann transform. Expressing things in terms of Gaussian measure rather than Lebesgue measure also permits one to let the dimension tend to infinity, allowing, for example, an infinite-dimensional version of the Fourier transform (the Fourier-Wiener transform) and of the Hermite expansion (the chaos expansion, a basic tool in stochastic analysis).

There have been various constructions over the years that allow similar developments on manifolds other than Euclidean space. This article summarizes one set of such constructions, in which Euclidean space is replaced by a Lie group and the Gaussian measure on Euclidean space is replaced by a heat kernel measure on the Lie group. The results that I will describe here had their beginning with work of L. Gross in 1993, and the theory has been developed over the last decade by Gross and many other authors. I will describe a number of these results as well as several open problems.

The original results of Gross were intimately related to stochastic analysis, specifically, analysis on loop groups. Although most of the results described in this survey can be understood purely non-probabilistically, several other connections with stochastic analysis have emerged. (See Section 4.) In another direction, results involving heat kernels on groups have provided surprising new examples in geometric quantization, including the phenomenon of "quantization commuting with reduction". (See Section 3 and Section 4.2.) The methods described here have also been used to give new insights into the quantization of 2-dimensional Yang-Mills theory. (See Section 4.2.) Many of the results here involve natural "creation and annihilation operators"; a challenging open problem is to understand these results in terms of some variant of the canonical commutation relations and the Stonevon Neumann theorem. (See Section 2.5.) It is my hope that this survey will draw attention to some of the interesting open problems in this area.

In this introduction I will summarize some of the results for the Euclidean case. I will concentrate on three different Hilbert spaces and the natural unitary isomorphisms among them. For a more detailed exposition of some of these results see [H6], F. Although the terminology comes from the mathematical physics literature, these constructions are also an important part of harmonic analysis. All of the constructions depend on a positive parameter $t$, which may be interpreted as Planck's constant.

Three Hilbert spaces are involved. The first is the position Hilbert space, $L^{2}\left(\mathbb{R}^{d}, \rho_{t}\right)$. Here $\rho_{t}$ is the normalized Gaussian measure given by

$$
d \rho_{t}(x)=(2 \pi t)^{-d / 2} e^{-x^{2} / 2 t} d x .
$$

Here $x^{2}=x_{1}^{2}+\cdots+x_{d}^{2}$. The use of Gaussian measure instead of Lebesgue measure is merely a convenience that simplifies many of the constructions described here. As long as the dimension $d$ remains finite, one can always translate all results back to the Lebesgue measure setting.

The second Hilbert space is the Segal-Bargmann space $\mathcal{H} L^{2}\left(\mathbb{C}^{d}, \mu_{t}\right)$. Here $\mathcal{H} L^{2}\left(\mathbb{C}^{d}, \mu_{t}\right)$ denotes the space of entire holomorphic functions on $\mathbb{C}^{d}$ that are square-integrable with respect to the measure $\mu_{t}$ given by

$$
d \mu_{t}(z)=(\pi t)^{-d} e^{-|z|^{2} / t} d z .
$$

Here $d z$ denotes $2 d$-dimensional Lebesgue measure on $\mathbb{C}^{d}$. 
The third Hilbert space is what I will call the Fock space. (The Segal-Bargmann space is also sometimes called "Fock space".) I will give here a slightly unorthodox description of the Fock space, which will be convenient later. Let $S\left(\mathbb{R}^{d}\right)$ denote the symmetric algebra over $\mathbb{R}^{d}$. The symmetric algebra is just the tensor algebra $T\left(\mathbb{R}^{d}\right)$, modulo the two-sided ideal $I$ generated by elements of the form $X \otimes Y-Y \otimes X$, with $X, Y \in \mathbb{R}^{d}$. Now let $I^{0}\left(\mathbb{R}^{d}\right)$ denote the space of complex-valued linear functionals on $T\left(\mathbb{R}^{d}\right)$ that are zero on the ideal $I$. So $I^{0}\left(\mathbb{R}^{d}\right)$ is just the complex-valued dual space to the symmetric algebra $S\left(\mathbb{R}^{d}\right)=T\left(\mathbb{R}^{d}\right) / I$. A typical element $\xi$ of $I^{0}\left(\mathbb{R}^{d}\right)$ is a sum

$$
\xi=\xi_{0}+\xi_{1}+\xi_{2}+\cdots,
$$

where each $\xi_{n}$ is a symmetric element of the dual of $\left(\mathbb{R}^{d}\right)^{\otimes n}$. We make no restriction at the moment on the behavior of the $\xi_{n}$ 's as $n$ tends to infinity.

Now let $X_{1}, \cdots, X_{d}$ be an orthonormal basis for $\mathbb{R}^{d}$ with respect to the standard inner product. Define a norm $\|\cdot\|_{n}$ on the dual of $\left(\mathbb{R}^{d}\right)^{\otimes n}$ by setting

$$
\left\|\xi_{n}\right\|_{n}^{2}=\sum_{k_{1}, \cdots, k_{n}=1}^{d}\left|\xi_{n}\left(X_{k_{1}} \otimes \cdots \otimes X_{k_{n}}\right)\right|^{2} .
$$

This norm is independent of the choice of orthonormal basis for $\mathbb{R}^{d}$. Next define a norm $\|\cdot\|_{t}$ on $I^{0}\left(\mathbb{R}^{d}\right)$ by setting

$$
\|\xi\|_{t}^{2}=\sum_{n=0}^{\infty} \frac{t^{n}}{n !}\left\|\xi_{n}\right\|_{n}^{2},
$$

where the $\xi_{n}$ 's are as in (1.3). Then the Fock space $I_{t}^{0}\left(\mathbb{R}^{d}\right)$ is defined as

$$
I_{t}^{0}\left(\mathbb{R}^{d}\right)=\left\{\xi \in I^{0}\left(\mathbb{R}^{d}\right) \mid\|\xi\|_{t}<\infty\right\} .
$$

There are natural unitary maps between each pair of Hilbert spaces described above. These unitary maps allow one to transfer problems from one space to another; each of the three spaces has advantages for certain types of problems. The position Hilbert space is how we are most accustomed to thinking about things. The Segal-Bargmann space is a sort of "phase space" Hilbert space that incorporates simultaneously information about a function and its Fourier transform, or in quantum-mechanical lingo, information about the position and the momentum of a particle. The Fock space encodes the decomposition of a function into "excited states" for a quantum harmonic oscillator. In the infinite-dimensional theory $(d \rightarrow \infty)$, the Fock space encodes the decomposition into states describing a fixed number of particles. Besides the position Hilbert space, Segal-Bargmann space, and Fock space there is also the momentum Hilbert space, which is obtained from the position Hilbert space by means of the Fourier transform. I will not discuss further the momentum Hilbert space in this survey.

These three Hilbert spaces, and the isomorphisms between them, can be understood in terms of the "creation and annihilation operators" and the "canonical commutation relations". This point of view is discussed in Section 2.5 and, among other places, in [H6]. 
First, between the position Hilbert space and the Segal-Bargmann space we have the Segal-Bargmann transform. For $f \in L^{2}\left(\mathbb{R}^{d}, \rho_{t}\right)$ we define the SegalBargmann transform $B_{t} f$ of $f$ by

$$
B_{t} f(z)=(2 \pi t)^{-d / 2} \int_{\mathbb{R}^{d}} e^{-(z-x)^{2} / 2 t} f(x) d x, \quad z \in \mathbb{C}^{d}
$$

Here $(z-x)^{2}=\left(z_{1}-x_{1}\right)^{2}+\cdots+\left(z_{d}-x_{d}\right)^{2}$. It is not hard to see that for each $f \in$ $L^{2}\left(\mathbb{R}^{d}, \rho_{t}\right)$, the integral in (1.6) converges absolutely and the result is a holomorphic function of $z \in \mathbb{C}^{d}$. Note that if we restrict attention to $z \in \mathbb{R}^{d}$, then $B_{t} f(z)$ is just the convolution of $f$ with a Gaussian. This is just the solution of the heat equation at time $t$ with initial condition $f$. Thus we may write

$$
B_{t} f=\text { analytic continuation of } e^{t \Delta / 2} f \text {. }
$$

Here $e^{t \Delta / 2} f$ is a mnemonic for the solution of the heat equation with initial function $f$, and the analytic continuation is from $\mathbb{R}^{d}$ to $\mathbb{C}^{d}$ with $t$ fixed. My convention is that the Laplacian is a negative operator, so that $e^{t \Delta / 2}$ is the forward heat operator.

A theorem of Segal and Bargmann is the following.

Theorem 1 (Segal-Bargmann). For all $t>0$, the map $B_{t}$ is a unitary map of $L^{2}\left(\mathbb{R}^{d}, \rho_{t}\right)$ onto $\mathcal{H} L^{2}\left(\mathbb{C}^{d}, \mu_{t}\right)$.

See $[\mathrm{B}],[\mathrm{H} 6],[\mathrm{BSZ},[\mathrm{F}$ ) for more information.

Next we consider the Taylor map, a unitary map between the Segal-Bargmann space $\mathcal{H} L^{2}\left(\mathbb{C}^{d}, \mu_{t}\right)$ and the Fock space. Let us consider a more concrete description of the Fock space. Suppose $\xi=\xi_{0}+\xi_{1}+\cdots$ is an element of the Fock space $I_{t}^{0}$, as in (1.3). Then each $\xi_{n}$ is a complex-valued linear functional on $\left(\mathbb{R}^{d}\right)^{\otimes n}$. So if $X_{1}, \cdots, X_{d}$ is an orthonormal basis for $\mathbb{R}^{d}$, then $\xi_{n}$ is determined by the complex numbers

$$
\alpha_{k_{1}, \cdots, k_{n}}:=\xi_{n}\left(X_{k_{1}} \otimes \cdots \otimes X_{k_{n}}\right) .
$$

Since $\xi$ annihilates the ideal $I$, each $\xi_{n}$ must be a symmetric linear functional, so the numbers $\alpha_{k_{1}, \cdots, k_{n}}$ must be invariant under permutation of the indices $k_{1}, \cdots, k_{n}$. The norm of $\xi$ (as given in (1.5) ) may be expressed in terms of the $\alpha_{k_{1}, \cdots, k_{n}}$ 's as

$$
\|\xi\|_{t}^{2}=\sum_{n=0}^{\infty} \frac{t^{n}}{n !} \sum_{k_{1}, \cdots, k_{n}=1}^{d}\left|\alpha_{k_{1}, \cdots, k_{n}}\right|^{2} .
$$

Since the $\alpha$ 's are symmetric this may be rewritten in a way that involves only sets of indices with $k_{1} \leq \cdots \leq k_{n}$, with some additional combinatorial factors. However, for purposes of generalization to the group case, (1.8) is the most convenient way to write things.

So now given a function $F \in \mathcal{H} L^{2}\left(\mathbb{C}^{d}, \mu_{t}\right)$, we wish to produce an element of the Fock space $\xi$. We do this by setting

$$
\alpha_{k_{1}, \cdots, k_{n}}=\left(\frac{\partial^{n}}{\partial z_{k_{1}} \cdots \partial z_{k_{n}}} F\right)(0) .
$$

That is, the $\alpha$ 's are the Taylor coefficients of $F$ at the origin. Note that the $\alpha$ 's defined in this way are symmetric. Using a Taylor expansion of $F$, it is not difficult to see that

$$
\|F\|_{L^{2}\left(\mathbb{C}^{d}, \mu_{t}\right)}^{2}=\sum_{n=0}^{\infty} \frac{t^{n}}{n !} \sum_{k_{1}, \cdots, k_{n}=1}^{d}\left|\alpha_{k_{1}, \cdots, k_{n}}\right|^{2} .
$$


In the case $d=1$ this amounts to the statement that the functions $\left\{z^{n}\right\}_{n=0}^{\infty}$ form an orthogonal basis of $\mathcal{H} L^{2}\left(\mathbb{C}, \mu_{t}\right)$. Thus the "Taylor map" that associates to $F$ the collection of coefficients $\left\{\alpha_{k_{1}, \cdots, k_{n}}\right\}$ (as in (1.9) ) is an isometry of $\mathcal{H} L^{2}\left(\mathbb{C}^{d}, \mu_{t}\right)$ into the Fock space $I_{t}^{0}\left(\mathbb{R}^{d}\right)$. It is easily seen that the Taylor map is onto the Fock space. Inverting the Taylor map amounts to expanding $F$ in terms of the monomials $z_{1}^{n_{1}} \cdots z_{d}^{n_{d}}$-a Taylor series.

Finally, we consider the Hermite expansion, mapping from the position Hilbert space $L^{2}\left(\mathbb{R}^{d}, \rho_{t}\right)$ to the Fock space. This map is the composition of the SegalBargmann transform and the Taylor map. Since $F$ is holomorphic, its derivatives at zero may be computed by differentiating just in the real directions. Thus the Taylor coefficients of $B_{t} f$ (see (1.6) and (1.9)) may be expressed as

$$
\alpha_{k_{1}, \cdots, k_{n}}=\left(\frac{\partial^{n}}{\partial x_{k_{1}} \cdots \partial x_{k_{n}}} e^{t \Delta / 2} f\right)(0) .
$$

So we apply the heat equation to $f$, then compute all derivatives at the origin. In light of the two previous theorems, the map $f \rightarrow\left\{\alpha_{k_{1}, \cdots, k_{n}}\right\}$ given in (1.10) is an isometry of $L^{2}\left(\mathbb{R}^{d}, \rho_{t}\right)$ onto the Fock space. Inverting this map amounts to expanding $f$ in terms of the functions $H_{n_{1}, \cdots, n_{d}}$ such that

$$
B_{t}\left(H_{n_{1}, \cdots, n_{d}}\right)=z_{1}^{n_{1}} \cdots z_{d}^{n_{d}} .
$$

These functions are the Hermite polynomials given by

$$
H_{n_{1}, \cdots, n_{d}}=e^{-t \Delta / 2}\left(x_{1}^{n_{1}} \cdots x_{d}^{n_{d}}\right) .
$$

Here $e^{-t \Delta / 2}$ is the inverse heat operator, which is computed as a terminating power series. These polynomials may also be expressed in terms of derivatives of a Gaussian, as in Section 2.4.

Of course, there are many important aspects of these three Hilbert spaces that I have not discussed here. Most importantly, one should consider the natural operators on these spaces, namely, the creation and annihilation operators, and operators constructed from them. I discuss these operators in the group case in Section 2.5, with some comments about the simplifications that occur in the $\mathbb{R}^{d}$ case. There is also the important notion of coherent states, which I do not have space to discuss in this survey. (See [H6].) In the group case this notion has been pursued by T. Thiemann [T2].

It is a pleasure to thank those who have made corrections to this article: Maria Gordina, Wicharn Lewkeeratiyutkul, Jeffrey Mitchell, Stephen Sontz, and Matthew Stenzel.

\section{Three UnitARY MAPS FOR A COMPACT LiE GROUP}

Additional exposition of these results can be found in [G9].

2.1. Preliminaries. Let $K$ be a connected Lie group of compact type. A Lie group is said to be of compact type if it is locally isomorphic to some compact Lie group. Equivalently, a Lie group $K$ is of compact type if there exists an inner product on the Lie algebra of $K$ that is invariant under the adjoint action of $K$. So $\mathbb{R}^{d}$ is of compact type, being locally isomorphic to a $d$-torus, and every compact Lie group is of compact type. It can be shown that every connected Lie group of compact type is isomorphic to a product of $\mathbb{R}^{d}$ and a connected compact Lie group. So all of the constructions described here for Lie groups of compact type include as a special case the constructions for $\mathbb{R}^{d}$. On the other hand, all the new information 
(beyond the $\mathbb{R}^{d}$ case) is contained in the compact case. See [He, Chap. II, Sect. 6] (including Proposition 6.8) for information on Lie groups of compact type.

Let $\mathfrak{k}$ denote the Lie algebra of $K$. We fix once and for all an inner product $\langle\cdot, \cdot\rangle$ on $\mathfrak{k}$ that is invariant under the adjoint action of $K$. For example we may take $K=\mathrm{SU}(n)$, in which case $\mathfrak{k}=\mathrm{su}(n)$ is the space of skew matrices with trace zero. An invariant inner product on $\mathfrak{k}$ is $\langle X, Y\rangle=\operatorname{Re}\left[\operatorname{trace}\left(X^{*} Y\right)\right]$.

Now let $K_{\mathbb{C}}$ be the complexification of $K$. If $K$ is simply connected, then the complexification of $K$ is the unique simply connected Lie group whose Lie algebra $\mathfrak{k}_{\mathbb{C}}$ is $\mathfrak{k}+i \mathfrak{k}$. In general, $K_{\mathbb{C}}$ is defined by the following three properties. First, $K_{\mathbb{C}}$ should be a connected complex Lie group whose Lie algebra $\mathfrak{k}_{\mathbb{C}}$ is equal to $\mathfrak{k}+i \mathfrak{k}$. Second, $K_{\mathbb{C}}$ should contain $K$ as a closed subgroup (whose Lie algebra is $\mathfrak{k} \subset \mathfrak{k}_{\mathbb{C}}$ ). Third, every homomorphism of $K$ into a complex Lie group $H$ should extend to a holomorphic homomorphism of $K_{\mathbb{C}}$ into $H$. The complexification of a connected Lie group of compact type always exists and is unique.

Example 2. If $K=\mathbb{R}^{d}$, then $K_{\mathbb{C}}=\mathbb{C}^{d}$. If $K=\mathrm{SU}(n)$, then $K_{\mathbb{C}}=\mathrm{SL}(n ; \mathbb{C})$. If $K=\mathrm{SO}(n)$, then $K_{\mathbb{C}}=\mathrm{SO}(n ; \mathbb{C})$. In the first two examples, $K$ and $K_{\mathbb{C}}$ are simply connected. In the last example, neither $K$ nor $K_{\mathbb{C}}$ is simply connected.

We have the following structure theorem for Lie groups of compact type. This result is a modest strengthening of Corollary 2.2 of $[\mathrm{Dr}$ and allows all the relevant results for Lie groups of compact type to be reduced to two cases, the compact case and the $\mathbb{R}^{d}$ case.

Proposition 3. Suppose that $K$ is a connected Lie group of compact type, with a fixed Ad-invariant inner product on its Lie algebra $\mathfrak{k}$. Then there exists a decomposition $K=K_{1} \times K_{2}$, where $K_{1}$ is compact and $K_{2}$ is isomorphic to $\mathbb{R}^{d}$, and such that the associated Lie algebra decomposition $\mathfrak{k}=\mathfrak{k}_{1}+\mathfrak{k}_{2}$ is orthogonal.

2.2. The Segal-Bargmann transform for $K$. The Ad- $K$-invariant inner product on $\mathfrak{k}$ determines a bi-invariant Riemannian metric on $K$. Let $\Delta_{K}$ be the (nonpositive) Laplacian associated to this metric. Let $d x$ denote Haar measure on $K$, normalized to coincide with Riemannian volume measure. Then let $\rho_{t}$ denote the heat kernel (at the identity) on $K$, i.e. the solution to

$$
\frac{d \rho}{d t}=\frac{1}{2} \Delta_{K} \rho_{t}
$$

subject to the initial condition

$$
\lim _{t \downarrow 0} \int_{K} f(x) \rho_{t}(x) d x=f(e)
$$

for all continuous functions $f$ of compact support. We also let $\rho_{t}$ denote the heat kernel measure

$$
d \rho_{t}(x):=\rho_{t}(x) d x .
$$

If $K=\mathbb{R}^{d}$ (with the standard metric), then $\rho_{t}$ is nothing but the Gaussian measure (1.1). The following result is proved in [H1, Sect. 2].

Proposition 4. For all $t>0$ the function $\rho_{t}$ has a unique analytic continuation from $K$ to $K_{\mathbb{C}}$.

Thus we may define the Segal-Bargmann transform for $K$ by analogy to the $\mathbb{R}^{d}$ case. 
Definition 5. Let $\mathcal{H}\left(K_{\mathbb{C}}\right)$ denote the space of holomorphic functions on $K_{\mathbb{C}}$. Then for each $t>0$ define a map $B_{t}: L^{2}\left(K, \rho_{t}\right) \rightarrow \mathcal{H}\left(K_{\mathbb{C}}\right)$ by

$$
B_{t} f(g)=\int_{K} \rho_{t}\left(g x^{-1}\right) f(x) d x, \quad g \in K_{\mathbb{C}},
$$

where on the right $\rho_{t}\left(g x^{-1}\right)$ refers to the analytically continued heat kernel.

It is not obvious but true that the integral defining $B_{t} f$ is always convergent and that the result is holomorphic as a function of $g$. In the compact case there is no problem, and in the $\mathbb{R}^{d}$ case it is a straightforward calculation.

Note that the restriction of $B_{t} f$ to $K$ is just the convolution of $\rho_{t}$ with $f$. Since $\rho_{t}$ is the heat kernel, this means that the restriction of $B_{t} f$ to $K$ satisfies the heat equation, with initial condition $f$. Thus we may alternatively write

$$
B_{t} f=\text { analytic continuation of } e^{t \Delta_{K} / 2} f,
$$

where $e^{t \Delta_{K} / 2} f$ is shorthand for the solution of the heat equation given by the convolution of $\rho_{t}$ with $f$. The analytic continuation is in the space variable, from $K$ to $K_{\mathbb{C}}$, with the time parameter $t$ fixed.

We now construct an appropriate heat kernel measure on $K_{\mathbb{C}}$. We extend the inner product on $\mathfrak{k}$ to a real-valued inner product on $\mathfrak{k}_{\mathbb{C}}$ given by

$$
\left\langle X_{1}+i Y_{1}, X_{2}+i Y_{2}\right\rangle=\left\langle X_{1}, X_{2}\right\rangle+\left\langle Y_{1}, Y_{2}\right\rangle .
$$

Then there is a unique left-invariant Riemannian metric on $K_{\mathbb{C}}$ given by this inner product at the identity. Let $\Delta_{K_{\mathbb{C}}}$ be the Laplacian for this metric. We now define $\mu_{t}$ to be the heat kernel (at the identity) on $K_{\mathbb{C}}$, that is, the solution to

$$
\frac{d \mu}{d t}=\frac{1}{4} \Delta_{K_{\mathbb{C}}} \mu_{t}
$$

subject to the initial condition

$$
\lim _{t \downarrow 0} \int_{K_{\mathbb{C}}} f(g) \mu_{t}(g) d g=f(e)
$$

for all continuous functions $f$ with compact support. Here $d g$ is the (bi-invariant) Haar measure on $K_{\mathbb{C}}$, normalized to coincide with Riemannian volume measure. We also let $\mu_{t}$ denote the associated heat kernel measure

$$
d \mu_{t}(g):=\mu_{t}(g) d g .
$$

We are now ready to state the main theorem about the Segal-Bargmann transform for $K$.

Theorem 6. For all $t>0$ the map $B_{t}$ given in Definition 5 is a unitary map of $L^{2}\left(K, \rho_{t}\right)$ onto $\mathcal{H} L^{2}\left(K_{\mathbb{C}}, \mu_{t}\right)$, where $\mathcal{H} L^{2}\left(K_{\mathbb{C}}, \mu_{t}\right)$ denotes the space of holomorphic functions on $K_{\mathbb{C}}$ that are square-integrable with respect to $\mu_{t}$.

This is Theorem 1' of [H1]. (More precisely, [H1] proves the compact case. The case of general compact-type groups can be reduced to the compact case and the $\mathbb{R}^{d}$ case due to Segal and Bargmann.) A sketch of the proof of this result and the other main theorems is given in Section 5 . 
2.3. The Taylor map. Let $\left\{X_{k}\right\}_{k=1}^{\operatorname{dim} \mathfrak{k}}$ be an orthonormal basis for $\mathfrak{k}$. We think of each $X_{k}$ as a left-invariant vector field on $K_{\mathbb{C}}$, satisfying

$$
X_{k} f(g)=\left.\frac{d}{d s}\right|_{s=0} f\left(g e^{s X_{k}}\right), \quad g \in K_{\mathbb{C}} .
$$

Then the norm of a function $F \in \mathcal{H} L^{2}\left(K_{\mathbb{C}}, \mu_{t}\right)$ can be expressed as follows in terms of its derivatives at the identity.

Theorem 7. Let $K$ be a connected Lie group of compact type, and $K_{\mathbb{C}}$ the complexification of $K$. Let $\left\{X_{k}\right\}_{k=1}^{\operatorname{dim} \mathfrak{k}}$ be an orthonormal basis for $\mathfrak{k}$ (with respect to a fixed Ad-K-invariant inner product). Then for all $F \in \mathcal{H} L^{2}\left(K_{\mathbb{C}}, \mu_{t}\right)$

$$
\int_{K_{\mathbb{C}}}|F(g)|^{2} \mu_{t}(g) d g=\sum_{n=0}^{\infty} \frac{t^{n}}{n !} \sum_{k_{1}, \cdots, k_{n}=1}^{\operatorname{dim} \mathfrak{k}}\left|\left(X_{k_{1}} X_{k_{2}} \cdots X_{k_{n}} F\right)(e)\right|^{2} .
$$

This theorem and the succeeding one are consequences of Theorem $1^{\prime}$ of [H1] and Proposition 2.4 of [Hi1. They are stated explicitly in [Dr, Cor. 1.17]. At a formal level the above identity is easily obtained by expanding the left side in powers of t. (See Section 5.2.) This result expresses the norm of $F$ in terms of its "Taylor coefficients", namely, the left-invariant derivatives of $F$ at the identity.

We wish to understand what possible collections of Taylor coefficients can arise, so as to have an algebraic description of our Hilbert space, comparable to the Fock symmetric tensor model in the $\mathbb{R}^{d}$ case. There are two restrictions on the Taylor coefficients. First there is an algebraic condition: the commutation relations of the Lie algebra impose certain linear relations among the derivatives $\left(X_{k_{1}} X_{k_{2}} \cdots X_{k_{n}} F\right)(e)$. For example, $X_{j} X_{k}$ is equal to $X_{k} X_{j}$ plus $\left[X_{j}, X_{k}\right]$, which is a linear combination of the $X_{l}$ 's. So a certain linear combination of $X_{j} X_{k}, X_{k} X_{j}$, and the $X_{l}$ 's is zero, and this linear relation is necessarily reflected in the derivatives of $F$ at $e$. Similar relations exist among higher-order derivatives. Second there is an analytic condition: the weighted sum of the squares of the derivatives, as in Theorem 7 , must be finite. If $K$ (or equivalently $K_{\mathbb{C}}$ ) is simply connected, these two conditions are sufficient.

Theorem 8. Suppose $\left\{\alpha_{k_{1}, \cdots, k_{n}}\right\}$ is a collection of complex numbers satisfying the algebraic relations associated to the Lie algebra $\mathfrak{k}$ and such that

$$
\sum_{n=0}^{\infty} \frac{t^{n}}{n !} \sum_{k_{1}, \cdots, k_{n}=1}^{\operatorname{dim} \mathfrak{k}}\left|\alpha_{k_{1}, \cdots, k_{n}}\right|^{2}<\infty .
$$

Suppose also that $K$ is simply connected. Then there exists a unique function $F \in$ $\mathcal{H} L^{2}\left(K_{\mathbb{C}}, \mu_{t}\right)$ such that $\left(X_{k_{1}} X_{k_{2}} \cdots X_{k_{n}} F\right)(e)=\alpha_{k_{1}, \cdots, k_{n}}$.

The basis-independent way of saying this is as follows. Consider the tensor algebra over $\mathfrak{k}, T(\mathfrak{k})$. Let $J$ be the two-sided ideal in $T(\mathfrak{k})$ generated by elements of the form

$$
X \otimes Y-Y \otimes X-[X, Y], \quad X, Y \in \mathfrak{k} .
$$

Then the quotient algebra $U(\mathfrak{k}):=T(\mathfrak{k}) / J$ is the universal enveloping algebra of $\mathfrak{k}$. Let $T(\mathfrak{k})^{*}$ be the complex dual space, i.e. the set of all linear maps of $T(\mathfrak{k})$ into $\mathbb{C}$. This may be expressed as

$$
\sum_{n=0}^{\infty}\left(\mathfrak{k}^{*}\right)^{\otimes n} \quad(\text { strong direct sum })
$$


where $\mathfrak{k}^{*}$ is the complex dual space of $\mathfrak{k}$. There is a natural norm $\|\cdot\|_{n}$ on $\left(\mathfrak{k}^{*}\right)^{\otimes n}$ given as in (1.4) above.

Now a general element $\alpha$ of $T(\mathfrak{k})^{*}$ decomposes as $\xi=\xi_{0}+\xi_{1}+\xi_{2}+\cdots$, with $\xi_{n} \in\left(\mathfrak{k}^{*}\right)^{\otimes n}$. Define a $\left(t\right.$-dependent) norm $\|\cdot\|_{t}$ on $T(\mathfrak{k})^{*}$ by

$$
\|\xi\|_{t}^{2}=\sum_{n=0}^{\infty} \frac{t^{n}}{n !}\left\|\xi_{n}\right\|_{n}^{2} .
$$

Since we impose no condition on the sequence $\xi_{n} \in\left(\mathfrak{k}^{*}\right)^{\otimes n}$, the norm $\|\xi\|_{t}$ may take the value $+\infty$.

Definition 9. Let $J^{0}$ be the set of linear functionals $\xi$ in $T(\mathfrak{k})^{*}$ such that $\xi$ is zero on the ideal $J$. Equivalently, elements $\xi \in J^{0}$ may be thought of as linear functionals on the universal enveloping algebra $T(\mathfrak{k}) / J$.

Let $J_{t}^{0}$ be the set of elements $\xi \in J^{0}$ such that $\|\xi\|_{t}<\infty$.

Now as usual we may think of the universal enveloping algebra $U(\mathfrak{k})=T(\mathfrak{k}) / J$ as the algebra of left-invariant differential operators on $K$. Given a function $F \in$ $\mathcal{H} L^{2}\left(K_{\mathbb{C}}, \mu_{t}\right)$ we wish to define a linear functional on $U(\mathfrak{k})$ - that is, an element of $J^{0}$-called the Taylor map of $F$. This is given by

$$
\text { Taylor }(F)(\alpha)=(\alpha F)(e), \quad \alpha \in U(\mathfrak{k}) .
$$

On the right we are (implicitly) restricting $F$ to $K$, then applying the differential operator $\alpha$, then evaluating at the identity. One could more generally apply leftinvariant derivatives on $K_{\mathbb{C}}$ to $F$ and evaluate at the identity. But since $F$ is holomorphic, these could all be reduced to derivatives in the $K$ directions.

We may now rephrase the previous two results in basis-independent terms as follows.

Theorem 10. The Taylor map $F \rightarrow$ Taylor $(F)$ is an isometric map of $\mathcal{H} L^{2}\left(K_{\mathbb{C}}, \mu_{t}\right)$ into $J_{t}^{0}$. If $K$ is simply connected, then the Taylor map takes $\mathcal{H} L^{2}\left(K_{\mathbb{C}}, \mu_{t}\right)$ onto $J_{t}^{0}$.

The proof of isometricity of the Segal-Bargmann transform relies on the fact that the inner product on $\mathfrak{k}$ is Ad- $K$-invariant. This is why the real group $K$ must be of compact type (i.e. admit an Ad-invariant inner product). But an examination of the proof of the isometricity of the Taylor map (say as given in $[\mathrm{Dr}]$ ) shows that this does not use the Ad-invariance of the inner product. In fact, as shown by Driver and Gross, this isomorphism can be generalized to arbitrary complex Lie groups, as I will now describe. I revert at this stage to basis-dependent notation.

Let $G$ be an arbitrary connected complex Lie group with Lie algebra $\mathfrak{g}$ and complex dimension $d$. Let $J: \mathfrak{g} \rightarrow \mathfrak{g}$ denote the complex structure on $\mathfrak{g}$. Fix an arbitrary Hermitian inner product $(\cdot, \cdot)$ on $\mathfrak{g}$. Then $\langle\cdot, \cdot\rangle:=\operatorname{Re}(\cdot, \cdot)$ is a realvalued inner product on $\mathfrak{g}$, which is invariant under $J$. Let $X_{1}, \cdots, X_{d}$ be a basis for $\mathfrak{g}$ as a complex vector space that is orthonormal with respect to $(\cdot, \cdot)$. Then $X_{1}, \cdots, X_{d}, J X_{1}, \cdots, J X_{d}$ is an orthonormal basis for $\mathfrak{g}$ as a real vector space. We regard $X_{1}, \cdots, X_{d}, J X_{1}, \cdots, J X_{d}$ as left-invariant vector fields on $G$. For a general smooth function $f,(J X) f$ is not the same as $i(X f)$, although these two quantities are equal if $f$ is holomorphic. Now define

$$
\Delta_{G}=\sum_{k=1}^{d}\left[X_{k}^{2}+\left(J X_{k}\right)^{2}\right] .
$$


We call this the Laplacian for $G$. If $G$ is unimodular, then $\Delta_{G}$ coincides with the Laplace-Beltrami operator for $G$ with respect to the left-invariant Riemannian metric determined by $\langle\cdot, \cdot\rangle$.

Let $\mu_{t}$ denote the "heat kernel" for $\Delta_{G}$, i.e. the solution of

$$
\frac{d \mu}{d t}=\frac{1}{4} \Delta_{G} \mu_{t}(g)
$$

subject to the initial condition

$$
\lim _{t \downarrow 0} \int_{G} f(g) \mu_{t}(g) d g=f(e),
$$

for all continuous functions $f$ with compact support, where $d g$ is a fixed rightinvariant Haar measure on $G$. The use of a left-invariant Laplacian and a rightinvariant Haar measure is so that $\Delta_{G}$ will be self-adjoint in $L^{2}(G, d g)$.

Theorem 11. If $F \in \mathcal{H} L^{2}\left(G, \mu_{t}(g) d g\right)$, then

$$
\int_{G}|F(g)|^{2} \mu_{t}(g) d g=\sum_{n=0}^{\infty} \frac{t^{n}}{n !} \sum_{k_{1}, \cdots, k_{n}=1}^{d}\left|\left(X_{k_{1}} X_{k_{2}} \cdots X_{k_{n}} F\right)(e)\right|^{2} .
$$

Suppose that $G$ is simply connected. Suppose also that $\alpha_{k_{1}, \cdots, k_{n}}$ is a collection of complex numbers satisfying the algebraic relations associated to the Lie algebra $\mathfrak{g}$ and such that

$$
\sum_{n=0}^{\infty} \frac{t^{n}}{n !} \sum_{k_{1}, \cdots, k_{n}=1}^{d}\left|\alpha_{k_{1}, \cdots, k_{n}}\right|^{2}<\infty
$$

Then there exists a unique $F \in \mathcal{H} L^{2}\left(G, \mu_{t}(g) d g\right)$ such that $\left(X_{k_{1}} X_{k_{2}} \cdots X_{k_{n}} F\right)(e)=$ $\alpha_{k_{1}, \cdots, k_{n}}$.

These results are Theorems 2.5 and 2.6 of [DG]. M. Gordina has obtained similar results for certain infinite-dimensional complex Lie groups [Go1, [Go2].

The Taylor expansion gives the following useful pointwise bound on functions in $\mathcal{H} L^{2}\left(G, \mu_{t}(g) d g\right)$.

Theorem 12. Let $G$ and $\mu_{t}$ be as in Theorem 11, For $g \in G$, let $|g|$ denote the distance from the identity to $g$ with respect to the left-invariant Riemannian metric on $G$ determined by the inner product $\langle\cdot, \cdot\rangle$ on $\mathfrak{g}$. Then for all $F \in \mathcal{H} L^{2}\left(G, \mu_{t}(g) d g\right)$ we have

$$
|F(g)|^{2} \leq\|F\|_{L^{2}\left(G, \mu_{t}\right)}^{2} e^{|g|^{2} / t} .
$$

This result follows from Corollary 3.10 and Remark 3.11 of [DG], with $k=0$. In the case $G=K_{\mathbb{C}}$ (with an Ad- $K$-invariant inner product on $\mathfrak{k}_{\mathbb{C}}$ ), this result was obtained previously in [Dr, Cor. 5.5 and Thm. 5.7]. (Compare [B Eq. (1.7)].) In the case $G=K_{\mathbb{C}}$ it is possible to give exponentially better bounds than those in Theorem 12. (See Section 3.3 and [H3.) Nevertheless the bounds in Theorem 12 are very useful and play an important role in the proofs in $[\mathrm{Dr}]$ and $[\mathrm{DG}]$, as well as in [HS], Go1], Go2].

Open Problem 1. Suppose that $G, \mu_{t}$ are as in Theorem 11 Assuming that $G$ has a faithful finite-dimensional representation, prove that the matrix entries of the finite-dimensional holomorphic representations span a dense subspace of $\mathcal{H} L^{2}\left(G, \mu_{t}(g) d g\right)$. 
Open Problem 2. Suppose that $G, \mu_{t}$ are as in Theorem 11 Prove that for all $\varepsilon>0, \mathcal{H} L^{2}\left(G, \mu_{t+\varepsilon}(g) d g\right)$ is dense in $\mathcal{H} L^{2}\left(G, \mu_{t}(g) d g\right)$.

If $G=K_{\mathbb{C}}$ and the inner product on $\mathfrak{g}$ is invariant under the adjoint action of $K$, then both problems have been solved in the affirmative, as a consequence of the "averaging lemma" [H1 Lem. 11]. But even the case $G=K_{\mathbb{C}}$ with an arbitrary Hermitian inner product on $\mathfrak{g}$ is open.

2.4. The Hermite expansion. Note that since each vector field $X_{k}$ in Theorem [7] is in $\mathfrak{k}$, all the derivatives on the right in that theorem involve only the values of $F$ on $K$. (Since $F$ is holomorphic, all of the derivatives at the identity, in any direction, can be computed in terms of derivatives in the $K$-directions.) Suppose now that $F$ is the Segal-Bargmann transform of some function $f$ in $L^{2}\left(K, \rho_{t}\right)$. Then the restriction of $F$ to $K$ is just $e^{t \Delta_{K} / 2} f$ (see (2.1)). So combining the isometricity of the Segal-Bargmann transform and the isometricity of the Taylor map we get the following result.

Theorem 13. Let $K$ be a connected Lie group of compact type, and $\left\{X_{k}\right\}_{k=1}^{\operatorname{dim} \mathfrak{k}}$ an orthonormal basis for $\mathfrak{k}$ with respect to a fixed Ad-K-invariant inner product. Then for all $f \in L^{2}\left(K, \rho_{t}\right)$

$$
\int_{K}|f(x)|^{2} \rho_{t}(x) d x=\sum_{n=0}^{\infty} \frac{t^{n}}{n !} \sum_{k_{1}, \cdots, k_{n}=1}^{\operatorname{dim} \mathfrak{k}}\left|\left(X_{k_{1}} X_{k_{2}} \cdots X_{k_{n}} e^{t \Delta_{K} / 2} f\right)(e)\right|^{2} .
$$

Suppose that $\left\{\alpha_{k_{1}, \cdots, k_{n}}\right\}$ is a collection of complex numbers satisfying the algebraic relations associated to the Lie algebra $\mathfrak{k}$ and such that

$$
\sum_{n=0}^{\infty} \frac{t^{n}}{n !} \sum_{k_{1}, \cdots, k_{n}=1}^{\operatorname{dim} \mathfrak{k}}\left|\alpha_{k_{1}, \cdots, k_{n}}\right|^{2}<\infty
$$

Suppose also that $K$ is simply connected. Then there exists a unique $f \in L^{2}\left(K, \rho_{t}\right)$ such that $\left(X_{k_{1}} X_{k_{2}} \cdots X_{k_{n}} e^{t \Delta_{K} / 2} f\right)(e)=\alpha_{k_{1}, \cdots, k_{n}}$ for all $k_{1}, \cdots, k_{n}$.

This result is a consequence of [G2, Thm. 2.1]. (See also [G3, G4.) The result as given here first appears in [Hi1, Props. 2.4 and 2.5]. See also [Dr, Thm. 1.4] and $[\mathrm{Hi} 2$.

The Hermite expansion leads naturally to a notion of Hermite functions on $K$, namely the functions $H_{k_{1}, \cdots, k_{n}}$ on $K$ such that

$$
\left(X_{k_{1}} X_{k_{2}} \cdots X_{k_{n}} e^{t \Delta_{K} / 2} f\right)(e)=\left\langle H_{k_{1}, \cdots, k_{n}}, f\right\rangle_{L^{2}\left(K, \rho_{t}\right)} .
$$

A straightforward calculation establishes the following Rodriguez-type formula for the Hermite functions

$$
H_{k_{1}, \cdots, k_{n}}=(-1)^{n} \frac{X_{k_{n}} \cdots X_{k_{1}} \rho_{t}}{\rho_{t}} .
$$

In the case $K=\mathbb{R}^{d}$ these are the usual Hermite polynomials. In the compact case these functions have been studied by J. Mitchell. In [M1] he shows that after a suitable re-scaling, the generalized Hermite polynomials converge as $t$ tends to zero to the ordinary Hermite polynomials. In [M2] he develops a small- $t$ asymptotic expansion for the Hermite functions, in terms of ordinary Hermite polynomials. 
2.5. Creation and annihilation operators. Gross G2 originally described the Hermite expansion in terms of the action of the relevant "annihilation operators". In this section we will consider the annihilation operators in each of our three unitarily related Hilbert spaces, as well as their adjoints, the creation operators. The creation and annihilation operators are also called raising and lowering operators.

Starting with a connected Lie group $K$ of compact type, we have considered three Hilbert spaces: the position Hilbert space $L^{2}\left(K, \rho_{t}\right)$, the Segal-Bargmann space $\mathcal{H} L^{2}\left(K_{\mathbb{C}}, \mu_{t}\right)$, and the Fock-type "tensor" space $J_{t}^{0}$ (the space of linear functionals on $T(\mathfrak{k}) / J$ with finite norm). Each of these three spaces has a naturally defined set of creation and annihilation operators, labeled by elements of the Lie algebra $\mathfrak{k}$. At some risk of confusion, I am going to use the same symbols for the creation and annihilation operators in each of the three Hilbert spaces. So in each Hilbert space we will have a family of annihilation operators $a_{X}$, labeled by elements $X$ of the Lie algebra $\mathfrak{k}$, and also a corresponding family of creation operators $a_{X}^{*}$. In each case the creation operators are defined to be the adjoints of the corresponding annihilation operators: $a_{X}^{*}:=\left(a_{X}\right)^{*}$ (in case this is not already evident from the notation). In addition to the creation and annihilation operators there is a vacuum state (or ground state), a unique (up to a constant) element of the Hilbert space that is annihilated by all the annihilation operators. In each case the annihilation operators satisfy the commutation relations of the Lie algebra: $\left[a_{X}, a_{Y}\right]=a_{[X, Y]}$. As a consequence the creation operators satisfy $\left[a_{X}^{*}, a_{Y}^{*}\right]=-a_{[X, Y]}^{*}$.

The position Hilbert space $L^{2}\left(K, \rho_{t}\right)$. We think of the Lie algebra elements $X$ as left-invariant vector fields. We define the annihilation operators to be simply these vector fields:

$$
a_{X} f(x)=X f(x)=\left.\frac{d}{d s}\right|_{s=0} f\left(x e^{s X}\right) .
$$

The vacuum state is the constant function $\mathbf{1}$. We then define the creation operators to be the adjoints of the left-invariant vector fields, computed with respect to the inner product for $L^{2}\left(K, \rho_{t}\right)$. Explicitly we have

$$
a_{X}^{*}=-X-X\left(\log \rho_{t}\right) .
$$

Here the second term is a multiplication operator, by the derivative of the logarithm of the heat kernel. In the $\mathbb{R}^{d}$ case, $X\left(\log \rho_{t}\right)$ is just a linear function.

The Segal-Bargmann space $\mathcal{H} L^{2}\left(K_{\mathbb{C}}, \mu_{t}\right)$. Here the annihilation operators are again defined to be left-invariant vector fields, but now regarded as acting on $K_{\mathbb{C}}$ instead of $K$. To avoid confusion, we now let $J: \mathfrak{k}_{\mathbb{C}} \rightarrow \mathfrak{k}_{\mathbb{C}}$ denote the complex structure on $\mathfrak{k}_{\mathbb{C}}$. For a general smooth function $f$ on $K_{\mathbb{C}},(J X) f \neq i(X f)$. Indeed, $f$ is holomorphic if and only if $(J X) f=i(X f)$ for all $X \in \mathfrak{k}_{\mathbb{C}}$.

When acting on holomorphic functions the real vector field $X$ coincides with the holomorphic vector field $(X-i J X) / 2$. So in the Segal-Bargmann space we have

$$
\begin{aligned}
a_{X} F(g) & =\frac{1}{2}(X-i J X) F(g) \\
& =\left.\frac{1}{2} \frac{d}{d s}\left(F\left(g e^{s X}\right)-i F\left(g e^{s J X}\right)\right)\right|_{s=0} .
\end{aligned}
$$

These operators preserve the space of holomorphic functions. In the case $K_{\mathbb{C}}=$ $\mathbb{C}^{d}$ these holomorphic vector fields are just linear combinations of the operators 
$\partial / \partial z_{k}:=\left(\partial / \partial x_{k}-i \partial / \partial y_{k}\right) / 2$. In all cases the vacuum state is the constant function 1.

The creation operators (defined to be the adjoints of the annihilation operators) can be computed as Toeplitz operators, namely,

$$
a_{X}^{*} F=P_{t}\left(\phi_{X, t} F\right)
$$

where

$$
\phi_{X, t}=-\frac{1}{2}(X+i J X)\left(\log \mu_{t}\right) .
$$

Here $P_{t}$ is the orthogonal projection operator from $L^{2}\left(K_{\mathbb{C}}, \mu_{t}\right)$ onto the holomorphic subspace $\mathcal{H} L^{2}\left(K_{\mathbb{C}}, \mu_{t}\right)$. So we take the holomorphic function $F$, multiply by the typically non-holomorphic function $-(X+i J X)\left(\log \mu_{t}\right) / 2$, and then project back into the holomorphic subspace. In verifying this formula for $a_{X}^{*}$ one uses integration by parts and the Cauchy-Riemann equations for $F$ in the form $(J X) F=i(X F)$.

In the case $K_{\mathbb{C}}=\mathbb{C}^{d}$, the operators $(1 / 2)(X+i J X)$ are just linear combinations of the operators $\partial / \partial \bar{z}_{k}$. We compute that $-\partial \log \mu_{t} / \partial \bar{z}_{k}=z_{k} / t$. Since in this case the logarithmic derivative is holomorphic, the projection is unnecessary and the creation operator is just multiplication by $z_{k} / t$.

The "Fock space" $J_{t}^{0}$. Recall that elements of $J_{t}^{0}$ are linear functionals on the universal enveloping algebra $T(\mathfrak{k}) / J$ with finite norm. The annihilation operators in $J_{t}^{0}$ are defined as follows. Given $X \in \mathfrak{k}$ and $\xi \in J_{t}^{0}$, we define

$$
a_{X} \xi(\alpha)=\xi(\alpha X), \quad \alpha \in T(\mathfrak{k}) / J .
$$

That is, $a_{X}$ is the adjoint of right-multiplication by $X$ in the universal enveloping algebra $T(\mathfrak{k}) / J$. The vacuum state in $J_{t}^{0}$ is the linear functional $\phi_{0}$ that picks out the coefficient of the identity for each $\alpha \in T(\mathfrak{k}) / J$. The creation operators are the adjoints of the annihilation operators, computed with respect to the natural $(t$ dependent) inner product on $J_{t}^{0}$. If one uses the inner product to identify $J_{t}^{0}$ with a completion of the enveloping algebra $T(\mathfrak{k}) / J$, then the creation operators are given simply as right-multiplication by $X$.

I should emphasize that in all cases the creation and annihilation operators are unbounded. So these operators are defined only on some dense domain in the corresponding Hilbert space. Although understanding the domains of these operators can be tricky, I will not concern myself with domain issues here.

Let us assume now that $K$ (and therefore also $K_{\mathbb{C}}$ ) is simply connected. Then between any two of these spaces we have a natural unitary map. It is almost immediately evident that these unitary maps intertwine the annihilation operators. For example, the Segal-Bargmann transform $B_{t}$ satisfies

$$
B_{t} a_{X}=a_{X} B_{t}, \quad X \in \mathfrak{k} .
$$

This is because the Laplacian and the heat operator are bi-invariant and therefore commute with the left-invariant vector fields $X$. The unitarity of $B_{t}$ then implies that

$$
B_{t} a_{X}^{*}=a_{X}^{*} B_{t}, \quad X \in \mathfrak{k} .
$$

So the Segal-Bargmann transform "intertwines" both the creation and the annihilation operators. It also takes the vacuum to the vacuum, as is easily verified. Similar statements apply to the Taylor map and therefore also to the Hermite expansion map. 
It is not hard to see that $B_{t}$ is the unique map (up to constant) satisfying (2.2). Similar statements apply to the Taylor map and to the Hermite expansion. Indeed this intertwining property was the way in which Gross originally described the Hermite expansion in G2, Thm. 2.1]. See also Corollary 2.11 and Proposition 7.5 of G9.

Open Problem 3. Explain the existence of these unitary intertwining maps.

This is a vaguely stated problem, which I now explain in more detail. I will also discuss below one possible solution to this problem. Suppose we have a Hilbert space $H$ and a collection of "annihilation operators" $\left\{a_{X}\right\}_{X \in \mathfrak{k}}$ such that the following two properties hold.

(A) For all $X, Y \in \mathfrak{k},\left[a_{X}, a_{Y}\right]=a_{[X, Y]}$.

(B) There is a unique (up to a constant) unit vector $\psi_{0} \in H$ such that $a_{X} \psi_{0}=0$ for all $X$.

Now suppose we have another such space with its own annihilation operators and vacuum state. Under what conditions does there exist a unitary map between the two Hilbert spaces that intertwines the vacuum and the annihilation operators? The preceding discussion says that if $K$ is simply connected, then such unitary intertwining maps do exist between any two of the three spaces $L^{2}\left(K, \rho_{t}\right), \mathcal{H} L^{2}\left(K_{\mathbb{C}}, \mu_{t}\right)$, and $J_{t}^{0}$. On the other hand, since $(\mathrm{A})$ and $(\mathrm{B})$ do not impose any restriction on the inner product on $H$, one clearly cannot expect a unitary intertwining map to exist in general. Thus it is natural to look for some additional properties, beyond (A) and (B), that hold in these three spaces and that could "explain" the existence of the unitary intertwining maps.

It is reasonable to take as our "target" Hilbert space the Fock space $J_{t}^{0}$, since this space does not depend on whether $K$ is simply connected or not and since it is constructed algebraically using just the commutation relations (A). So we may ask: Under what conditions on the space $H$ and the operators $a_{X}$ does there exist a unitary map $U$ from $H$ to $J_{t}^{0}$ such that $U \psi_{0}=\phi_{0}$ and such that $U a_{X}=a_{X} U$ for all $X$ ? The goal is to find general conditions (in addition to (A) and (B)) that would guarantee the existence of such a map. If such conditions could be seen to hold in $L^{2}\left(K, \rho_{t}\right)$ and $\mathcal{H} L^{2}\left(K_{\mathbb{C}}, \mu_{t}\right)$, then these conditions would "explain" why the annihilation operators in those two spaces are unitarily equivalent to the annihilation operators in $J_{t}^{0}$.

Suppose that there were an isometric map $U$ from $H$ into $J_{t}^{0}$ such that $U \psi_{0}=\phi_{0}$ and such that $U a_{X}=a_{X} U$ for all $X \in \mathfrak{k}$. Then the adjoint map $U^{*}: J_{t}^{0} \rightarrow H$ would have to satisfy

$$
U^{*}\left(a_{X_{1}}^{*} \cdots a_{X_{n}}^{*} \phi_{0}\right)=a_{X_{1}}^{*} \cdots a_{X_{n}}^{*} \psi_{0} .
$$

So we have no choice as to what $U^{*}$ does on the "excited states" $a_{X_{1}}^{*} \cdots a_{X_{n}}^{*} \phi_{0}$, which span a dense subspace of $J_{t}^{0}$. This suggests that we simply define a map $U^{*}$ by (2.4) and then define $U$ to be $\left(U^{*}\right)^{*}$. We must then answer two questions. First, under what conditions on the system $\left(H,\left\{a_{X}\right\}\right)$ will the map $U$ defined by means of (2.4) be isometric? Second, under what conditions will $U$ map onto $J_{t}^{0}$ ?

In the case $K=\mathbb{R}^{d}$, we do have conditions that will guarantee affirmative answers to both questions, namely the canonical commutation relations:

$$
t\left[a_{X}, a_{Y}^{*}\right]=\langle X, Y\rangle I
$$


This relation is in addition to the commutative case of (A), which tells us that $\left[a_{X}, a_{Y}\right]=\left[a_{X}^{*}, a_{Y}^{*}\right]=0$. The relation (2.5) can be verified in each of the three Hilbert spaces $L^{2}\left(\mathbb{R}^{d}, \rho_{t}\right), \mathcal{H} L^{2}\left(\mathbb{C}^{d}, \mu_{t}\right)$, and $I_{t}^{0}$. The Stone-von Neumann theorem tells us (assuming irreducibility and some domain conditions) that there is a unique intertwining map between any two Hilbert spaces satisfying the canonical commutation relations (2.5) and $\left[a_{X}, a_{Y}\right]=\left[a_{X}^{*}, a_{Y}^{*}\right]=0$. Concretely, one can use the canonical commutation relations inductively to compute the inner products of different excited states. One gets a definite answer independent of the particular representation of the canonical commutation relations, which shows that the map defined in (2.4) is isometric (indeed unitary assuming irreducibility). (See [H6], [RS.)

So in the $\mathbb{R}^{d}$ case the canonical commutation relations are the information we need, in addition to (A) and (B), to guarantee that $U$ in (2.4) is unitary. However, for general Lie groups of compact type we do not have any simple analog of the canonical commutation relations. Among the annihilation operators we have the relation $\left[a_{X}, a_{Y}\right]=a_{[X, Y]}$, and among the creation operators we have $\left[a_{X}^{*}, a_{Y}^{*}\right]=$ $-a_{[X, Y]}^{*}$. But between a creation operator and an annihilation operator things are not so simple. In $L^{2}\left(K, \rho_{t}\right)$ we can compute that $\left[a_{X}, a_{Y}^{*}\right]=-[X, Y]-X Y\left(\log \rho_{t}\right)$. In the $\mathbb{R}^{d}$ case $[X, Y]=0$ and $X Y\left(\log \rho_{t}\right)$ is a constant, and we recover the canonical commutation relations. In general, the second derivatives of the logarithm of the heat kernel are non-constant. Indeed $\left[a_{X}, a_{Y}^{*}\right]$ is neither a multiple of the identity nor any other operator that can be expressed in terms of the creation and annihilation operators - it is just another algebraically unrelated operator. Higher commutators, such as $\left[a_{X},\left[a_{Y}, a_{Z}^{*}\right]\right]$, involve higher derivatives of the heat kernel, which again are not expressible in terms of the commutators we already have. There is therefore no simple analog of (2.5) for general Lie groups of compact type.

There is, however, another relation that does hold in general and might be a partial substitute for the missing canonical commutation relations. Let $\left\{X_{k}\right\}$ be an orthonormal basis for $\mathfrak{k}$, and let $a_{k}:=a_{X_{k}}$ be the associated annihilation operators. Then I claim that the identity operator can be expanded as

$$
I=\sum_{n=0}^{\infty} \frac{t^{n}}{n !} \sum_{k_{1}, \cdots, k_{n}=1}^{\operatorname{dim} \mathfrak{k}} a_{k_{1}}^{*} \cdots a_{k_{n}}^{*}\left|\psi_{0}\right\rangle\left\langle\psi_{0}\right| a_{k_{n}} \cdots a_{k_{1}} .
$$

Here the $a_{k}$ 's are the annihilation operators in any one of the three Hilbert spaces $L^{2}\left(K, \rho_{t}\right), \mathcal{H} L^{2}\left(K_{\mathbb{C}}, \mu_{t}\right)$, or $J_{t}^{0}$, and $\psi_{0}$ is the vacuum state in that Hilbert space. The operator $\left|\psi_{0}\right\rangle\left\langle\psi_{0}\right|$ is the orthogonal projection onto the vacuum state. It is not too hard to verify this relation at least formally in each of the three cases, using the techniques of Section 5 .

My conjecture is that if we have a Hilbert space $H$ satisfying (A) and (B) above, and also satisfying (2.6) (and suitable domain conditions), then there will exist a unique isometric embedding of $H$ into $J_{t}^{0}$ (given by (2.4)) which intertwines the annihilation operators and the vacuum.

If this is correct, then one would seek further conditions under which $H$ maps onto $J_{t}^{0}$. One necessary condition for surjectivity is that there be no extra relations among the annihilation operators (besides (A)). That is, we need that the algebra generated by the annihilation operators be isomorphic to the full universal enveloping algebra. Unfortunately, this condition is not sufficient to distinguish between $L^{2}\left(K, \rho_{t}\right)$ with $K$ simply connected (which maps onto $\left.J_{t}^{0}\right)$ and $L^{2}\left(K, \rho_{t}\right)$ with $K$ 
not simply connected (which maps to a proper subspace of $J_{t}^{0}$ ). So some stronger condition is needed.

\section{The $K$-invariant Segal-Bargmann transform}

3.1. The transform. Recall that $\mu_{t}$ is the heat kernel (at the identity) on $K_{\mathbb{C}}$. Now consider the function $\nu_{t}$ on $K_{\mathbb{C}}$ given by

$$
\nu_{t}(g):=\int_{K} \mu_{t}(g x) d x .
$$

This function is just the heat kernel (at the identity coset) on the symmetric space $K_{\mathbb{C}} / K$, regarded as a $K$-invariant function on $K_{\mathbb{C}}$. We also consider the associated measure

$$
d \nu_{t}(g):=\nu_{t}(g) d g .
$$

Theorem 14. For all $t>0$ the map $C_{t}$ given by

$$
C_{t} f=\text { analytic continuation of } e^{t \Delta_{K} / 2} f
$$

is a unitary map of $L^{2}(K, d x)$ onto $\mathcal{H} L^{2}\left(K_{\mathbb{C}}, \nu_{t}\right)$.

This result (for the compact case) is Theorem 2 of [H1]. Note that the map $C_{t}$ is given by precisely the same formula as the transform $B_{t}$; only the measures on $K$ and $K_{\mathbb{C}}$ have changed.

In the $\mathbb{R}^{d}$ case, the transform $C_{t}$ is nothing but the usual Segal-Bargmann transform, with trivial differences of normalization. For example, comparing to Bargmann's transform $A$ we have simply (with $t=1$ as in [B] $)$

$$
C_{1} f(z)=c e^{-z^{2} / 4} A f\left(\frac{z}{\sqrt{2}}\right),
$$

where $c$ is a constant whose value is unimportant. In turn Bargmann's transform differs from the finite-dimensional version of Segal's [S3] essentially just by the "ground state transformation". In the general group case, $C_{t}$ and $B_{t}$ are "inequivalent". Nevertheless, in light of the above observations, we may say that in the $\mathbb{R}^{d}$ case both reduce to the classical Segal-Bargmann transform.

For certain problems the $C_{t}$ version of the transform for $K$ is preferable to the $B_{t}$ version, mainly because it is invariant in a natural way under the left and right action of $K$. This section describes results that are (except in the $\mathbb{R}^{d}$ case) specific to the $K$-invariant version of the Segal-Bargmann transform.

3.2. The connection with geometric quantization. In this section I summarize the results of a forthcoming paper H8. The main result is that both the Segal-Bargmann space $\mathcal{H} L^{2}\left(K_{\mathbb{C}}, \nu_{t}\right)$ and the associated Segal-Bargmann transform $C_{t}$ may be obtained in a natural way by means of geometric quantization. This generalizes a well-known example from the $\mathbb{R}^{d}$ case [Wo Sect. 9.5].

Geometric quantization gives a method for constructing a "quantum" Hilbert space from a symplectic manifold $(\mathcal{M}, \omega)$ (the "classical phase space"). In order to carry out the quantization $\mathcal{M}$ must satisfy a certain integrality condition, which is always satisfied if the symplectic form $\omega$ is exact, as it will be in the case we will discuss here. The quantization procedure depends on a parameter $\hbar$ (Planck's constant), which in our case is to be identified with the parameter $t$ in $C_{t}$. The quantization also depends on the choice of a "polarization". Roughly, a polarization is a choice of $d$ coordinates on the $2 d$-dimensional phase space $\mathcal{M}$, with the idea that the 
functions in our quantum Hilbert space will be independent of these $d$ variables. For example if $\mathcal{M}=\mathbb{R}^{2 d}$, then we may take the usual position and momentum variables $x_{1}, \cdots, x_{d}, p_{1}, \cdots, p_{d}$ and then consider functions that depend only on $x_{1}, \cdots, x_{d}$ and are independent of $p_{1}, \cdots, p_{d}$. (This is the vertical polarization.) In that case our Hilbert space will consist of functions of $x_{1}, \cdots, x_{d}$ : the position Hilbert space. Alternatively, one may consider complex variables $z_{1}, \cdots z_{d}, \bar{z}_{1}, \cdots, \bar{z}_{d}$ and then consider the functions that are "independent of $\bar{z}_{k}$ ", that is, holomorphic. (This is a complex polarization.) In that case our Hilbert space is the Segal-Bargmann space.

To be more precise, in geometric quantization the elements of the quantum Hilbert space are not functions, but rather sections of a certain complex line bundle with connection. The sections are required to be covariantly constant in the directions corresponding to the polarization. The Gaussian measure in the SegalBargmann space arises naturally out of the bundle constructions [Wo, Sect. 9.2]. (See [Wo] and [Ki] for general information on geometric quantization and polarizations.)

If one has two different polarizations on the same symplectic manifold, then there is a natural "pairing map" that maps between the two associated Hilbert spaces. This map is not unitary in general, but is in certain very special cases. The prime example is $\mathcal{M}=\mathbb{R}^{2 d}$ (with the standard symplectic form). If one uses the vertical polarization, then the resulting Hilbert space can be identified with $L^{2}\left(\mathbb{R}^{d}\right)$, the position Hilbert space. If one uses a complex polarization, then the resulting Hilbert space is the Segal-Bargmann space, with the parameter $t$ now playing the role of Planck's constant. In this case the pairing map between the two Hilbert spaces is just the Segal-Bargmann transform. In particular the pairing map is unitary in this case. (The exact form of the spaces and the transform depends on certain choices, namely the choice of trivializing sections of the relevant line bundles.)

I now wish to consider $\mathcal{M}=T^{*}(K)$, the cotangent bundle of a connected Lie group of compact type. As a cotangent bundle $T^{*}(K)$ has a canonical symplectic structure. I will describe in the next paragraph how $T^{*}(K)$ can be given a certain complex polarization, obtained by identifying $T^{*}(K)$ with $K_{\mathbb{C}}$. It turns out that the result of geometric quantization of $T^{*}(K)$ (with this complex polarization) can be identified precisely with the $K$-invariant form of the Segal-Bargmann space, $\mathcal{H} L^{2}\left(K_{\mathbb{C}}, \nu_{t}\right)$, provided that one includes the "half-form correction" in the quantization. Not only so, but the pairing map between the complex-polarized Hilbert space and the vertically-polarized Hilbert space turns out to coincide exactly with the $K$-invariant Segal-Bargmann transform. In particular, the pairing map is unitary in this case. These results are remarkable because the Segal-Bargmann space and Segal-Bargmann transform are defined in terms of heat kernels, whereas geometric quantization seemingly has nothing to do with heat kernels or the heat equation.

I will now describe these results in greater detail. The polar decomposition for $K_{\mathbb{C}}$ gives a diffeomorphism between $K_{\mathbb{C}}$ and the cotangent bundle $T^{*}(K)$. This diffeomorphism is natural from at least two different points of view, as explained in [H3] and [H7]. Explicitly, the diffeomorphism is as follows. Identify $T^{*}(K)$ with $K \times \mathfrak{k}^{*}$ by means of left-translation, and then with $K \times \mathfrak{k}$ by means of the inner 
product on $\mathfrak{k}$. Then consider the map $\Phi$ from $K \times \mathfrak{k}$ to $K_{\mathbb{C}}$ given by

$$
\Phi(x, Y)=x e^{i Y}, \quad x \in K, Y \in \mathfrak{k} .
$$

Then $\Phi$ is a diffeomorphism whose inverse is essentially the polar decomposition for $K_{\mathbb{C}}$.

This diffeomorphism allows us to transport the complex structure of $K_{\mathbb{C}}$ to $T^{*}(K)$. The resulting complex structure on $T^{*}(K)$ fits together with the canonical symplectic structure so as to make $T^{*}(K)$ into a Kähler manifold. Thus in the language of geometric quantization we have on $K_{\mathbb{C}}$ a Kähler polarization. This Kähler structure on $T^{*}(K)$ has a global Kähler potential given by

$$
\kappa(x, Y)=|Y|^{2} \text {. }
$$

This Kähler potential satisfies $\operatorname{Im} \bar{\partial} \kappa=\theta$, where $\theta$ is the canonical 1-form on $T^{*}(K)$, given by $\theta=\Sigma p_{k} d q_{k}$ in the usual sort of local coordinates. This complex structure and the associated Kähler potential come from the work of Guillemin and Stenzel and of Lempert and Szóke. (See [GStenz, Sect. 5] and [LS, Cor. 5.5].)

We now follow the prescription of geometric quantization. First we construct a prequantum line bundle $L$, which is a Hermitian line bundle with connection whose curvature is equal to $\omega / \hbar$, where $\omega$ is the canonical symplectic form on $T^{*}(K)$. In our case $\omega=d \theta$ is exact, and so we may choose our line bundle $L$ to be topologically trivial; in fact we just take $L=T^{*}(K) \times \mathbb{C}$, with covariant derivative given by $\nabla_{X}=X+\frac{1}{i \hbar} \theta(X)$ (see [Wo, Eq. (8.4.4)]).

Next we use the Kähler polarization on $T^{*}(K)$ to define the notion of polarized sections of $L$. These are the sections that are covariantly constant in the $\bar{z}$-directions. Since we are using a Kähler polarization, $L$ obtains the structure of a holomorphic line bundle, and the polarized sections are precisely the holomorphic sections. Since our line bundle is trivial, the sections are just functions. The holomorphic sections, however, are not the same as holomorphic functions. Rather, the holomorphic sections are functions of the form

$$
s=\exp (-\kappa / 2 \hbar) F
$$

where $F$ is a holomorphic function and $\kappa$ is the Kähler potential (3.2).

Finally, we consider the half-form correction. This means that we consider the canonical bundle for $T^{*}(K)$ (relative to the Kähler polarization), namely, the holomorphic line bundle whose holomorphic sections are holomorphic $d$-forms on $T^{*}(K)$. (Here $d=\operatorname{dim} K=\frac{1}{2} \operatorname{dim} T^{*}(K)$.) This bundle has a natural Hermitian structure given by $|\alpha|^{2}=\left(i^{d} \alpha \wedge \bar{\alpha}\right) / \omega^{d}$. In our case, the canonical bundle is holomorphically trivial, since thinking of $T^{*}(K)$ as $K_{\mathbb{C}}$ we have a nowhere-vanishing

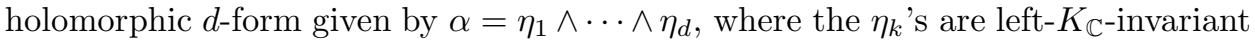
holomorphic 1-forms. We then consider a bundle $\delta$, which is a square root of the canonical bundle. In this case we may take $\delta$ to be a holomorphically trivial bundle whose square is isomorphic to the canonical bundle. Then $\delta$ inherits a Hermitian structure from the canonical bundle, defined by requiring that if $s$ is a section of $\delta$, then $|s|=|s \otimes s|^{1 / 2}$.

So then we consider the holomorphic line bundle $L \otimes \delta$, which has a natural Hermitian structure, since both $L$ and $\delta$ do. The quantum Hilbert space is then the space of holomorphic sections $s$ of $L \otimes \delta$ that are square-integrable with respect 
to the natural volume measure $\omega^{d}$ on $T^{*}(K)$. For such a section $s$ we define $\|s\|$ by

$$
\|s\|^{2}=\int_{T^{*}(K)}|s|^{2} \omega^{d}
$$

In our case, both $L$ and $\delta$ are holomorphically trivial, with trivializing sections $\exp (-\kappa / 2 \hbar)$ for $L$ and $\sqrt{\alpha}$ for $\delta$. Thus every holomorphic section is of the form

$$
s=\exp (-\kappa / 2 \hbar) F \otimes \sqrt{\alpha}
$$

where $F$ is a holomorphic function. We may then identify the section $s$ with the function $F$, with the norm as follows:

$$
\|F\|^{2}=\int_{T^{*}(K)}|s|^{2} \omega^{d}=\int_{T^{*}(K)}|F|^{2} e^{-\kappa / \hbar}\left|\frac{i^{d} \alpha \wedge \bar{\alpha}}{\omega^{d}}\right|^{1 / 2} \omega^{d} .
$$

Proposition 15. For each $\hbar>0$ there exists a constant $c_{\hbar}$ so that under the identification of $T^{*}(K)$ with $K_{\mathbb{C}}$ as above we have

$$
e^{-\kappa / \hbar}\left|\frac{i^{d} \alpha \wedge \bar{\alpha}}{\omega^{d}}\right|^{1 / 2} \omega^{d}=c_{\hbar} \nu_{\hbar}(g) d g
$$

This is to be compared with Section 7 of [H4], in which a similar calculation was performed, but without inclusion of the half-form correction. I am grateful to Dan Freed, who suggested to me that the discrepancy in [H4 might be accounted for by the half-form correction. Recall that in this subsection we are identifying the parameter $t$ in $C_{t}$ with $\hbar$.

To summarize, the $K$-invariant Segal-Bargmann space $\mathcal{H} L^{2}\left(K_{\mathbb{C}}, \nu_{\hbar}(g) d g\right.$ ) (up to an irrelevant overall constant) may be obtained by geometric quantization using a complex polarization and the half-form correction.

It may also be shown that the $K$-invariant form of the Segal-Bargmann transform coincides (up to an overall constant) with the pairing map of geometric quantization. In particular, the pairing map is (a multiple of) a unitary map in this case. More precisely, computing a formula for the pairing map similar to Equation (9.5.3) of [Wo, one sees that the pairing map coincides with the inverse Segal-Bargmann transform as given in $\mathrm{H2}$. Details of these results will appear in the forthcoming paper $\mathrm{H} 8$.

Open Problem 4. Explain why the pairing map of geometric quantization is unitary in this case.

This is the only case I know of, besides examples on $\mathbb{R}^{2 d}$, in which a pairing map is unitary. In examples on $\mathbb{R}^{2 d}$ the Stone-von Neumann theorem can explain why various pairing maps are unitary. In cases where the Stone-von Neumann theorem is inapplicable, there seems to be no reason to expect unitarity. The only non-trivial example besides the one discussed in this section where unitarity has been examined is one involving spheres investigated by J. Rawnsley $\underline{R}$, who found that the pairing map was not unitary. Thus it is surprising that the pairing map for $T^{*}(K)$ is unitary, and it seems that there must be some "explanation" for this phenomenon. (See the comments in Section 4.4 of [Ki].) 
3.3. Phase bounds. Suppose $f \in L^{2}(K, d x)$ has norm one. Then since the $K$ invariant Segal-Bargmann transform $C_{t}$ is unitary, $C_{t} f$ will be an element of norm one in $\mathcal{H} L^{2}\left(K_{\mathbb{C}}, \nu_{t}\right)$. This means by definition that

$$
\int_{K_{\mathbb{C}}}\left|C_{t} f(g)\right|^{2} \nu_{t}(g) d g=1
$$

Now in the last section we saw that the complex group $K_{\mathbb{C}}$ may be identified with the cotangent bundle $T^{*}(K)$. Using this identification of $K_{\mathbb{C}}$ with $T^{*}(K)$ (given in (3.1)), we may re-write (3.3) in terms of the canonical "phase volume measure" $\omega^{d}$ on $T^{*}(K)$. So (3.3) becomes

$$
\int_{K_{\mathbb{C}}}\left|C_{t} f(g)\right|^{2} \nu_{t}(g) \beta(g) \omega^{d}=1,
$$

where $\beta$ is the density (under our identification) of $\omega^{d}$ with respect to $d g$. It then seems natural to interpret the quantity

$$
\left|C_{t} f(g)\right|^{2} \nu_{t}(g) \beta(g)
$$

as the phase space probability density associated to the state $f$. Certainly this quantity is a probability density on phase space, that is, a positive function that integrates to one with respect to the natural phase space volume measure. Experience from the $\mathbb{R}^{d}$ case suggests that this is in some sense the most natural phase space density that can be associated to the state $f$. See $[\mathrm{H} 4,[\mathrm{H} 7]$ for further discussion. In the $\mathbb{R}^{d}$ case, the density in (3.5) is the "Husimi function" of the quantum state $f$.

If we are to interpret the quantity (3.5) as a phase space probability density, then we must take account of the uncertainty principle, which says roughly that states cannot be too concentrated in phase space. (The uncertainty principle is a "meta-theorem", which has many different precise formulations in different settings. See [FS].) If $C_{t} f$ were an arbitrary unit vector in $L^{2}\left(K_{\mathbb{C}}, \nu_{t}\right)$, we would have trouble with the uncertainty principle, because in that case (3.5) could be concentrated in an arbitrarily small region of the phase space. Fortunately, $C_{t} f$ is in the holomorphic subspace; this imposes very precise limits on the concentration of the density (3.5), as given in the following theorem.

Theorem 16. For any Lie group $K$ of compact type, there exist constants $a_{t}$ such that for all $f \in L^{2}(K, d x)$ with norm one we have

$$
\left|C_{t} f(g)\right|^{2} \nu_{t}(g) \beta(g) \leq a_{t}(2 \pi t)^{-d}
$$

for all points $g \in K_{\mathbb{C}}$. Here $d=\operatorname{dim} K$. The optimal constants $a_{t}$ tend to one exponentially fast as $t \rightarrow 0$. If $K=\mathbb{R}^{d}$, then we may take $a_{t}=1$.

This is Theorem 1 of [H3]. The proof of this result depends on the extremely good information that is available about the heat kernels on $K$ and on $K_{\mathbb{C}} / K$. The theorem says that the phase space probability density cannot be too big at any one point; since the density integrates to one, this means that it must be fairly spread out in phase space, in agreement with the uncertainty principle.

The bounds on the probability density are equivalent to bounds on holomorphic functions in $\mathcal{H} L^{2}\left(K_{\mathbb{C}}, \nu_{t}\right)$ (see Theorem 2 of [H3]). These bounds are exponentially better than the Driver-Gross bounds in Theorem[12. On the other hand, the DriverGross bounds hold in much greater generality and have dimension-independent constants. 
Open Problem 5. Explain why the constants in Theorem [16 tend to one exponentially fast.

One way of interpreting Theorem 16 is by saying that the normalized reproducing kernel, on the diagonal, is asymptotic to all orders to $(2 \pi t)^{-d}$. It is perhaps reasonable on general grounds (see for example the sort of results in [E]) to expect the reproducing kernel on the diagonal to be asymptotic to $(2 \pi t)^{-d}$ to leading order. Since in this case it is actually asymptotic to all orders, one may look for some special geometric property of this setting that could account for this.

3.4. Compact symmetric spaces. A compact symmetric space is a manifold of the form $K / H$ where $K$ is a compact Lie group and $H$ is a special sort of closed subgroup, namely, the fixed-point subgroup of an involution of $K$. Examples include the spheres $S^{n}$ and complex projective spaces $\mathbb{C P}^{n}$. M. Stenzel has generalized some of the results of [H1], H3] to this setting. One can get a Segal-Bargmann transform for $K / H$ simply by applying the Segal-Bargmann transform for $K$ and restricting to $H$-invariant functions, as in [H1 Sect. 11]. It is easily seen that this is the same as applying the heat operator for the symmetric space $K / H$ and then analytically continuing to $K_{\mathbb{C}} / H_{\mathbb{C}}$. The range Hilbert space will then be a Hilbert space of holomorphic functions on $K_{\mathbb{C}} / H_{\mathbb{C}}$.

The problem with this approach is that it does not give a nice description of the relevant measure on $K_{\mathbb{C}} / H_{\mathbb{C}}$. If we consider the $K$-invariant form $C_{t}$ of the transform, then the measure that arises on $K_{\mathbb{C}} / H_{\mathbb{C}}$ will be the push-forward under the quotient map of the measure $\nu_{t}$ on $K_{\mathbb{C}}$. Now, the measure $\nu_{t}$ is just the heat kernel measure for the non-compact symmetric space $K_{\mathbb{C}} / K$, viewed as a $K$-invariant measure on $K_{\mathbb{C}}$. However, it is not clear a priori whether the push-forward of $\nu_{t}$ under the quotient map is some sort of heat kernel measure.

Fortunately, M. Stenzel has solved this problem. He shows in St Thm. 3] that the Segal-Bargmann transform for $K / H$ is a unitary map onto the Hilbert space $\mathcal{H} L^{2}\left(K_{\mathbb{C}} / H_{\mathbb{C}}, \sigma_{t}\right)$. Here $\sigma_{t}$ is the heat kernel measure for the dual noncompact symmetric space to $K / H$. More precisely, Stenzel shows that $K_{\mathbb{C}} / H_{\mathbb{C}}$ is identifiable with $T^{*}(K / H)$. He then shows that each of the fibers of $T^{*}(K / H)$ may be identified with a certain non-compact symmetric space $G / H$, which is dual to $K / H$ in the sense of [He, Chap. V.2]. (Here $G$ is a certain subgroup of $K_{\mathbb{C}}$.) The measure $\sigma_{t}$ is then obtained by integrating over each fiber with respect to the heat kernel measure on $G / H$ and then integrating over $K / H$ with respect to the volume measure. For example, suppose that $K=S O(n+1)$ and $H=S O(n)$. Then $K / H=S^{n}$. In this case the non-compact symmetric space dual to $S^{n}$ is $n$-dimensional hyperbolic space $\mathbb{H}^{n}$. Stenzel shows how each fiber in $T^{*}\left(S^{n}\right)$ can be identified with $\mathbb{H}^{n}$. The measure $\sigma_{t}$ is then essentially the product of the heat kernel measure on $\mathbb{H}^{n}$ with the volume measure on $S^{n}$.

Stenzel's paper also extends the inversion formula of [H2] to the setting of compact symmetric spaces [St Thms. 1 and 2]. Indeed Stenzel first proves the inversion formula and then deduces the unitarity of the Segal-Bargmann from this. In the case of a 2-sphere, results related to those of Stenzel have been obtained independently and from a very different point of view by K. Kowalski and J. Rembieliński [KR.

Now, Stenzel does not prove that the measure $\sigma_{t}$ is the same as the push-forward from $K_{\mathbb{C}}$ of $\nu_{t}$. (He works directly at the level of the symmetric space and does not reduce things to the group case.) Nevertheless, $\sigma_{t}$ is the same as the push-forward 
from $K_{\mathbb{C}}$ of $\nu_{t}$. This is proved by F. Zhu [Z], using methods of M. Flensted-Jensen. The idea of [FJ] is that one can relate differential operators on $G / K$ to differential operators on $K_{\mathbb{C}} / K$; in particular it allows us to relate the heat equation on $G / K$ to the heat equation on $K_{\mathbb{C}} / K$. This is what we need to relate the push-forward of $\nu_{t}$ to $\sigma_{t}$.

Open Problem 6. Prove phase space bounds similar to those of Section 3.3 in which the group $K$ of compact type is replaced by a compact symmetric space.

It seems reasonable to expect that similar results (perhaps slightly weaker) will hold. However, the information about heat kernels that is needed is much harder to obtain in the general case than in the compact group case. That is, the dual pair of symmetric spaces $K$ and $K_{\mathbb{C}} / K$ is very special among all dual pairs. As a result there are special formulas for the heat kernel on these two spaces (see $\mathrm{H} 3$ ) that are not available for general pairs. Thus one has to work harder in the general case.

Open Problem 7. Determine whether or not the constructions of geometric quantization coincide with the Segal-Bargmann space and Segal-Bargmann transform for $K / H$. If not, explain why geometric quantization gives the same result as the "heat kernel" approach in the case of compact groups but not in the case of compact symmetric spaces.

I believe that in fact the geometric quantization constructions will not agree with those of Stenzel for general compact symmetric spaces. Again, the group case is special. It would be very nice to have a good geometrical explanation of why the two constructions agree in some cases but not in others.

Open Problem 8. Find a unitary Segal-Bargmann transform starting on a noncompact symmetric space.

That is, consider a system whose "position Hilbert space" is $L^{2}(X)$, where $X$ is a non-compact symmetric space such as hyperbolic space. If one simply tries to imitate the constructions from the compact case, one runs into very serious trouble; for example, the heat kernel simply does not have an analytic continuation of the expected sort. Although it is possible that there simply is no theorem here to be proved, I feel that this case should be understood better. Possibly we need a different way to think about the problem.

\section{ConneCtions With the theORY FOR Infinite-Dimensional LineaR SPACES}

4.1. Embedding the isomorphisms. The results described above for Lie groups had their beginning in the Hermite expansion for $K$, as described in Section 2.3. Theorem 13] was first proved (in a somewhat different form) by L. Gross in G2. However, the Hermite expansion for $K$ was not the main purpose of [G2]. Rather, the Hermite expansion was a fortuitous discovery along the path to a result in stochastic analysis that I discuss below. In G2 Gross thinks of $L^{2}\left(K, \rho_{t}\right)$ as a certain special subspace of the space of functions on an infinite-dimensional linear space. The generalized Hermite expansion for $K$ is actually then a special case of the ordinary Hermite expansion for an infinite-dimensional linear space (the $d \rightarrow \infty$ limit of the results in the introduction). Papers of Gross and Malliavin [GM], Hall and Sengupta [HS], Wren [Wr], and Driver and Hall [DH] have helped 
to understand better how the isomorphisms associated to a compact group relate to the corresponding isomorphisms for an infinite-dimensional linear space. For additional exposition of various of these relations see [G9] and also [G3], G4], [H7], [L2].

What happens is that each of the three Hilbert spaces associated to a compact Lie group can be embedded isometrically as a (very small) subspace of the corresponding Hilbert space for an infinite-dimensional linear space. Under this embedding the isomorphisms for the compact group match up with corresponding isomorphisms for the linear case as in Theorems 18 and 19 below. Thus each of the isomorphisms for the group case can be understood as a special case of the corresponding isomorphism for an infinite-dimensional linear space. It is helpful to have these two complementary ways of viewing the isomorphisms for $K$, either directly at the finite-dimensional level in a way that is merely analogous to the linear case, or actually as a special case of the isomorphisms for an infinite-dimensional linear space. For example, the pointwise bounds in Theorem 12 can be understood as a consequence of the embedding and the well-known bounds from the linear case.

Suppose as usual that $K$ is a connected Lie group of compact type and that $\mathfrak{k}$ is its Lie algebra, with a fixed Ad- $K$-invariant inner product. Then consider the real Hilbert space

$$
H_{\mathbb{R}}:=L^{2}([0,1] ; \mathfrak{k}),
$$

where the inner product on $H_{\mathbb{R}}$ is computed using the inner product on $\mathfrak{k}$. Consider also the associated complex Hilbert space

$$
H_{\mathbb{C}}:=L^{2}\left([0,1] ; \mathfrak{k}_{\mathbb{C}}\right)
$$

Then $H_{\mathbb{C}}=H_{\mathbb{R}}+i H_{\mathbb{R}}$. We wish to think of $H_{\mathbb{R}}$ as playing the role of $\mathbb{R}^{d}$ and $H_{\mathbb{C}}$ the role of $\mathbb{C}^{d}$, where now $d=\infty$.

We may attempt to construct a Gaussian measure, say $P_{t}$, on $H_{\mathbb{R}}$, which should be given heuristically by the formula

$$
d P_{t}(A)=b_{t} e^{-\|A\|^{2} / 2 t} \mathcal{D} A,
$$

where $\mathcal{D} A$ is the non-existent Lebesgue measure on $H_{\mathbb{R}}$ and $b_{t}$ is supposed to be a normalization constant. It is well known G1] that $P_{t}$ may be given a rigorous meaning as a probability measure on a suitable "extension" of $H_{\mathbb{R}}$, denoted $\bar{H}_{\mathbb{R}}$. For example, $\bar{H}_{\mathbb{R}}$ may be taken to be the space of $\mathfrak{k}$-valued distributions on $[0,1]$. The original space $H_{\mathbb{R}}$ is a set of $P_{t}$-measure zero inside $\bar{H}_{\mathbb{R}}$. The measure $P_{t}$ describes $\mathfrak{k}$-valued white noise (scaled by a factor of $\sqrt{t}$ ) or, equivalently, the derivative of scaled $\mathfrak{k}$-valued Brownian motion. Similarly we may construct a Gaussian measure $M_{t}$ on an extension $\bar{H}_{\mathbb{C}}$ of $H_{\mathbb{C}}$, where heuristically

$$
d M_{t}(Z)=c_{t} e^{-\|Z\|^{2} / t} \mathcal{D} Z \text {. }
$$

Then we have the position Hilbert space

$$
L^{2}\left(\bar{H}_{\mathbb{R}}, P_{t}\right)
$$

and the Segal-Bargmann space

$$
\mathcal{H} L^{2}\left(\bar{H}_{\mathbb{C}}, M_{t}\right)
$$

There are technical subtleties in the definition of the Segal-Bargmann space, which I will not discuss here. (See for example $\underline{\underline{\mathrm{Su}}}$.) Finally we have the dual of the 
symmetric algebra over $H_{\mathbb{R}}$, denoted

$$
I_{t}^{0}\left(H_{\mathbb{R}}\right)
$$

By a straightforward limit $d \rightarrow \infty$ we may extend the results for $\mathbb{R}^{d}$ to this setting, and so obtain unitary maps

$$
\begin{aligned}
& B_{t}: L^{2}\left(\bar{H}_{\mathbb{R}}, P_{t}\right) \rightarrow \mathcal{H} L^{2}\left(\bar{H}_{\mathbb{C}}, M_{t}\right) \\
& \text { Taylor }: \mathcal{H} L^{2}\left(\bar{H}_{\mathbb{C}}, M_{t}\right) \rightarrow I_{t}^{0}\left(H_{\mathbb{R}}\right) .
\end{aligned}
$$

The composition of the Segal-Bargmann transform and the Taylor map is the infinite-dimensional version of the Hermite expansion, which in this setting takes the form of an expansion into multiple stochastic integrals, as discussed below.

Now consider the holonomy map

$$
h: \bar{H}_{\mathbb{R}} \rightarrow K
$$

given by

$$
h(A)=\lim _{n \rightarrow \infty} e^{\int_{0}^{1 / n} A(\tau) d \tau} e^{\int_{1 / n}^{2 / n} A(\tau) d \tau} \cdots e^{\int_{(n-1) / n}^{1} A(\tau) d \tau} .
$$

It is possible to show that $h(A)$ is defined for $P_{t}$-almost every $A \in \bar{H}_{\mathbb{R}}$. More conventionally, $h(A)$ may be defined as the solution at time one of a certain stochastic differential equation, called the Itô map. (See (4.4) below.) Similarly we have

$$
h_{\mathbb{C}}: \bar{H}_{\mathbb{C}} \rightarrow K_{\mathbb{C}}
$$

given by

$$
h(Z)=\lim _{n \rightarrow \infty} e^{\int_{0}^{1 / n} Z(\tau) d \tau} e^{\int_{1 / n}^{2 / n} Z(\tau) d \tau} \cdots e^{\int_{(n-1) / n}^{1} Z(\tau) d \tau} .
$$

Formally $h_{\mathbb{C}}$ is just the analytic continuation of $h$ from $\bar{H}_{\mathbb{R}}$ to $\bar{H}_{\mathbb{C}}$. I will explain below a bit more the origin of these maps.

Proposition 17. For all $t>0$ the map

$$
\phi \rightarrow \phi \circ h
$$

is an isometric embedding of $L^{2}\left(K, \rho_{t}\right)$ into $L^{2}\left(\bar{H}_{\mathbb{R}}, P_{t}\right)$. For all $t>0$ the map

$$
\Phi \rightarrow \Phi \circ h_{\mathbb{C}}
$$

is an isometric embedding of $\mathcal{H} L^{2}\left(K_{\mathbb{C}}, \mu_{t}\right)$ into $\mathcal{H} L^{2}\left(\bar{H}_{\mathbb{C}}, M_{t}\right)$.

So we are embedding $L^{2}\left(K, \rho_{t}\right)$ into $L^{2}\left(\bar{H}_{\mathbb{R}}, P_{t}\right)$ as the space of functions of the form

$$
f(A)=\phi(h(A)),
$$

where $\phi$ is a function on $K$. The map $\phi \rightarrow \phi \circ h$ is isometric because the push-forward of the Gaussian measure $P_{t}$ under $h$ is precisely the heat kernel measure $\rho_{t}$. (In probabilistic language, the distribution of $h(A)$ with respect to $P_{t}$ is $\rho_{t}$.) Similarly we are embedding $\mathcal{H} L^{2}\left(K_{\mathbb{C}}, \mu_{t}\right)$ into $\mathcal{H} L^{2}\left(\bar{H}_{\mathbb{C}}, M_{t}\right)$ as the space of functions of the form $F(Z)=\Phi\left(h_{\mathbb{C}}(Z)\right)$, which is isometric because the push-forward of $M_{t}$ under $h_{\mathbb{C}}$ is $\mu_{t}$. A technical issue that must be resolved is to show that for $\Phi \in$ $\mathcal{H} L^{2}\left(K_{\mathbb{C}}, \mu_{t}\right), F=\Phi \circ h_{\mathbb{C}}$ is holomorphic in the appropriate sense on $\bar{H}_{\mathbb{C}}$. (See [HS. Sect. 2.5].) 
Theorem 18. Suppose that $f \in L^{2}\left(\bar{H}_{\mathbb{R}}, P_{t}\right)$ is of the form $f(A)=\phi(h(A))$, where $\phi$ is a function on $K$. Then

$$
B_{t} f(Z)=\Phi\left(h_{\mathbb{C}}(Z)\right)
$$

where $\Phi$ is the analytic continuation to $K_{\mathbb{C}}$ of $e^{t \Delta_{K} / 2} \phi$. In other words, on functions of the form $\phi \circ h$, the ordinary Segal-Bargmann transform for the infinitedimensional linear space $\bar{H}_{\mathbb{R}}$ reduces to the generalized Segal-Bargmann transform for the group $K$.

This result (in a slightly different form) is due to Gross and Malliavin [GM], Cor. 7.12]. See also [HS, Sect. 2.5].

Meanwhile, let us consider the infinite-dimensional version of the Hermite expansion for the space $L^{2}\left(\bar{H}_{\mathbb{R}}, P_{t}\right)$. This takes the form of an expansion into multiple stochastic integrals, the Wiener chaos expansion.

Theorem 19. Suppose that $f$ is a function on $\bar{H}_{\mathbb{R}}$ of the form $f(A)=\phi(h(A))$. Let $\xi \in J_{t}^{0}(\mathfrak{k})$ be the Hermite expansion of $\phi$ in the sense of Section 2.4. Then the Hermite expansion of $f$ is given by

$$
f(A)=\sum_{n=0}^{\infty} \int_{0}^{1} \int_{0}^{\tau_{n}} \cdots \int_{0}^{\tau_{2}} \xi_{n}\left(d a_{\tau_{1}} \otimes \cdots \otimes d a_{\tau_{n}}\right) .
$$

Here $a_{\tau}:=\int_{0}^{\tau} A_{\sigma} d \sigma$ is the Brownian motion associated to the white noise $A$, and the integrals are Itô stochastic integrals.

The situation is thus similar to that for the Segal-Bargmann transform. When applied to functions of the form $\phi \circ f$, the Hermite expansion for the linear space $\bar{H}_{\mathbb{R}}$ reduces to the generalized Hermite expansion for the compact group $K$. This result is a consequence of Theorems 2.4 and 2.5 of [G2, together with the explicit formula for the Hermite expansion as given in [Hi1, Prop. 2.4]. The result is obtained directly in Lemma 5.7 of $[\overline{\mathrm{DH}}]$. One may also compute the Taylor expansion of functions in $\mathcal{H} L^{2}\left(\bar{H}_{\mathbb{C}}, M_{t}\right)$ of the form $F(Z)=\Phi\left(h_{\mathbb{C}}(Z)\right)$, with similar results [DH, Lem. 5.8].

Note that in the chaos expansion of a general function in $L^{2}\left(\bar{H}_{\mathbb{R}}, P_{t}\right)$ the integrands $\xi_{n}$ would be functions of the variables $\tau_{1}, \cdots, \tau_{n}$. Here the integrands are just constants, and the elements $\xi_{n} \in\left(\mathfrak{k}^{*}\right)^{\otimes n}$ must fit together to define an element of $T(\mathfrak{k})^{*}$ that annihilates the ideal $J$.

Let me explain briefly the background to these results. Let $W(K)$ denote the continuous path group

$$
W(K)=\left\{\operatorname{maps} x:[0,1] \rightarrow K \mid x_{0}=e, x \text { is continuous }\right\} .
$$

There is a natural probability measure $w_{t}$ on $W(K)$, the Wiener measure, which describes Brownian motion in $K$. (The parameter $t$ is not the time variable for the Brownian motion, but rather a scaling factor that determines the diffusion rate of the Brownian motion.) Now let $\mathcal{L}(K)$ denote the finite-energy loop group:

$$
\mathcal{L}(K)=\left\{\operatorname{maps} l:[0,1] \rightarrow K \mid l_{0}=l_{1}=e, l \text { has one derivative in } L^{2}\right\} .
$$

There is a natural right action of $\mathcal{L}(K)$ on $W(K)$, given by $x \rightarrow x l$, with $x \in W(K)$, $l \in \mathcal{L}(K)$. This action leaves the Wiener measure quasi-invariant; that is, $d w_{t}(x l)$ is absolutely continuous with respect to $d w_{t}(x)$. 
Given the Wiener measure $w_{t}$, we may consider the Hilbert space $L^{2}\left(W(K), w_{t}\right)$. We may then consider the functions $f$ in $L^{2}\left(W(K), w_{t}\right)$ that are loop-invariant, that is, such that for all $l \in \mathcal{L}(K)$ we have

$$
f(x l)=f(x)
$$

for $w_{t}$-almost every $x \in W(K)$. One obvious class of loop-invariant functions is the class of endpoint functions, namely those of the form

$$
f(x)=\phi\left(x_{1}\right),
$$

where $\phi$ is a function on $K$. (Note that by the definition of $\mathcal{L}(K),(x l)_{1}=x_{1} l_{1}=$ $x_{1}$.)

Theorem 20. Every loop-invariant function in $L^{2}\left(W(K), w_{t}\right)$ is an endpoint function.

This result is a consequence of Theorem 2.5 of [G2]. The difficulty in proving this result is that we consider continuous paths but only finite-energy loops. The restriction to finite-energy loops is necessary to have quasi-invariance. Without quasi-invariance the notion of loop-invariance does not make sense, since the elements of $L^{2}\left(W(K), w_{t}\right)$ are not actually functions but rather equivalence classes of functions that are equal $w_{t}$-almost everywhere. On the other hand, we cannot restrict to finite-energy paths because they form a set of Wiener measure zero.

To prove this theorem Gross linearizes it, transferring the problem from the path group $W(K)$ to the linear space $\bar{H}_{\mathbb{R}}$. The transfer is accomplished by means of the Itô map, which is the measure-preserving map

$$
\theta:\left(\bar{H}_{\mathbb{R}}, P_{t}\right) \rightarrow\left(W(K), w_{t}\right)
$$

obtained by solving the Stratonovich stochastic differential equation

$$
d x_{\tau}=x_{\tau} \circ d a_{\tau}, \quad x_{0}=e,
$$

where as above $a_{\tau}:=\int_{0}^{\tau} A_{\sigma} d \sigma$ is the Brownian motion associated to the white noise $A$. Once the equation is solved we set $\theta(A)=x$. The holonomy map is nothing but the Itô map at time one:

$$
h(A)=\theta(A)_{1}, \quad A \in \bar{H}_{\mathbb{R}} .
$$

The Itô map induces a unitary map of $L^{2}\left(W(K), w_{t}\right)$ onto $L^{2}\left(\bar{H}_{\mathbb{R}}, P_{t}\right)$. Under this map, the endpoint functions in $L^{2}\left(W(K), w_{t}\right)$ go to functions of the holonomy in $L^{2}\left(\bar{H}_{\mathbb{R}}, P_{t}\right)$. Furthermore, one may use the Itô map to transfer the loop-group action on $W(K)$ to $\bar{H}_{\mathbb{R}}$. The resulting action is given by

$$
(l \cdot A)_{\tau}=l_{\tau} A_{\tau} l_{\tau}^{-1}-\frac{d l}{d \tau} l_{\tau}^{-1}, \quad l \in \mathcal{L}(K), A \in \bar{H}_{\mathbb{R}} .
$$

So Theorem 20 is equivalent to the statement that every function on $\bar{H}_{\mathbb{R}}$ that is invariant under the action (4.5) is of the form $f(A)=\phi(h(A))$, for some function $\phi$ on $K$. To prove this last statement, Gross proves that if $f$ is loop-invariant on $\bar{H}_{\mathbb{R}}$, then the chaos expansion of $f$ must be of the form (4.3), for some $\xi \in J_{t}^{0}$ G2. Thm. 5.1]. Then to construct the function $\phi$ on $K$, Gross essentially inverts the Hermite expansion for $K$ [G2 Sect. 8].

Of course, prior to $\mathrm{G} 2$, the Hermite expansion for $K$ was not known-it was discovered by Gross as a consequence of the investigations described in this section. For a simplified version of Gross's proof of Theorem [20] see Part II of [G9]. For a completely different subsequent proof, see Sadasue [Sa]. 
The original proof of the Hermite expansion for $K$ was in [G1] and involved the stochastic analysis described here. A purely finite-dimensional proof of the Hermite theorem for $K$ was then given by Hijab [Hi1], [Hi2] and Driver [Dr]. Meanwhile, Gross's results for the Hermite expansion motivated my own development of the Segal-Bargmann transform for $K$ in [H1. The proofs in [H1 are purely finitedimensional. Later, Gross and Malliavin in GM showed how to understand the Segal-Bargmann transform for $K$ in the way described here, connecting it with the infinite-dimensional linear theory.

P. Biane [Bi] has given a generalization of the Gross-Malliavin result to the setting of free probability theory. M. Gordina Go1, [Go2] has studied versions of the Driver-Gross theorem (Theorem 11) in the setting of infinite-dimensional Lie groups. T. Deck has given a group analog of the notion of Hida distributions in white noise analysis $[\mathrm{De}$.

4.2. The Yang-Mills interpretation. In $\mathrm{Wr}, \mathrm{K}$. Wren considers the problem of canonical quantization of Yang-Mills theory on a spacetime cylinder, using a method proposed by N. Landsman [L1]. (See also Chapter IV.3.8 of [L2].) Wren's calculations strongly suggest a close relationship between this Yang-Mills example and the $K$-invariant form of the Segal-Bargmann transform for a compact Lie group. The paper $[\mathrm{DH}]$ investigates this relationship further. (See also the expository paper [17.). Driver and I make a modification of the Gross-Malliavin results that fits with the desired Yang-Mills interpretation. (See also [ALMMT, [AHS], [Lo], T2] for other uses of the Segal-Bargmann transform for $K$ in connection with quantized gauge theories.)

The Yang-Mills problem considered is the simplest non-trivial case, namely YangMills theory on a spacetime cylinder. We take $K$ as our structure group. So time is a line, and space is a circle, which we think of as the interval $[0,1]$ with ends identified. We consider first classical Yang-Mills theory, using the temporal gauge. In the temporal gauge, the Yang-Mills equations may be considered as an infinite-dimensional Hamiltonian system whose configuration space is the space of square-integrable Lie algebra-valued 1 -forms on $[0,1]$. Since space is one-dimensional, we identify the configuration space with the space of square-integrable Lie algebra-valued functions. That is, our configuration space is $H_{\mathbb{R}}=L^{2}([0,1] ; \mathfrak{k})$. The associated phase space for the classical Yang-Mills theory is then the cotangent bundle of $H_{\mathbb{R}}$, which may be identified with $H_{\mathbb{C}}=L^{2}\left([0,1] ; \mathfrak{k}_{\mathbb{C}}\right)$.

The based gauge group for this theory is the set of maps $x$ of the space manifold $[0,1]$ into the structure group $K$, such that $x_{0}=x_{1}=e$. If (for technical reasons) we limit ourselves to maps with one derivative in $L^{2}$, then the based gauge group is nothing but the loop group $\mathcal{L}(K)$. The based gauge group acts on $H_{\mathbb{R}}$ in the usual way in gauge theory, namely by

$$
(l \cdot A)_{\tau}=l_{\tau} A_{\tau} l_{\tau}^{-1}-\frac{d l}{d \tau} l_{\tau}^{-1}, \quad l \in \mathcal{L}(K), A \in \bar{H}_{\mathbb{R}} .
$$

This is precisely the action (4.5) from the previous subsection, but now with a different interpretation. In the classical Yang-Mills theory, one is supposed to reduce by the action of the gauge group. This means that we replace the phase space $H_{\mathbb{C}}=T^{*}\left(H_{\mathbb{R}}\right)$ by $T^{*}\left(H_{\mathbb{R}} / \mathcal{L}(K)\right)$. So $T^{*}\left(H_{\mathbb{R}}\right)$ is the unreduced phase space and $T^{*}\left(H_{\mathbb{R}} / \mathcal{L}(K)\right)$ is the reduced or physical phase space. For a more detailed explanation of this reduction, see [DH], H7, [L2]. 
It is not difficult to show that two elements $A_{1}$ and $A_{2}$ of $H_{\mathbb{R}}$ are gauge-equivalent (i.e. there exists $l \in \mathcal{L}(K)$ with $\left.l \cdot A_{1}=A_{2}\right)$ if and only if $h\left(A_{1}\right)=h\left(A_{2}\right)$. Here $h(\cdot)$ is the holonomy map of (4.1), with the terminology motivated by this the YangMills interpretation. So the gauge equivalence classes are labeled by the holonomy $h(A) \in K$. This means that

$$
H_{\mathbb{R}} / \mathcal{L}(K) \cong K
$$

I will not discuss the dynamics of the classical Yang-Mills theory. (For the dynamics see $[\mathrm{DH}]$.) Instead I will consider quantizing the theory. The idea is to consider first quantizing the unreduced theory, obtaining a position Hilbert space, a Segal-Bargmann space, and a Segal-Bargmann transform corresponding to the configuration space $H_{\mathbb{R}}$. Having done that we then want to reduce appropriately by the gauge group $\mathcal{L}(K)$, which in light of (4.6) ought to give us a position Hilbert space, Segal-Bargmann space, and Segal-Bargmann transform for $K$. The set-up of the last section almost gives us what we want: we think of $L^{2}\left(\bar{H}_{\mathbb{R}}, P_{t}\right)$ as our position Hilbert space, and then we "reduce" by restricting to the functions in $L^{2}\left(\bar{H}_{\mathbb{R}}, P_{t}\right)$ that are invariant under the action of the loop group. By Theorem 20 (transferred to $\bar{H}_{\mathbb{R}}$ ), the loop-invariant functions are just functions of the holonomy, and by Theorem 18 these map under the Segal-Bargmann transform to functions of the complex holonomy.

Unfortunately, this set-up is not quite right from the point of view of quantization, because the loop group action on $L^{2}\left(\bar{H}_{\mathbb{R}}, P_{t}\right)$ is not unitary. That is, since the action of $\mathcal{L}(K)$ on $\bar{H}_{\mathbb{R}}$ leaves the measure $P_{t}$ only quasi-invariant, but not invariant, the $L^{2}$-norm of $f(l \cdot A)$ need not equal the $L^{2}$-norm of $f(A)$. Since in quantization theory the symmetries of the problem are supposed to act by unitary transformations, we have a problem.

To fix this problem, Driver and I considered as our position Hilbert space $L^{2}\left(\bar{H}_{\mathbb{R}}, P_{s}\right)$, where the variance parameter $s$ is large. Formally, as $s \rightarrow \infty, P_{s}$ converges to the (non-existent) Lebesgue measure on $\bar{H}_{\mathbb{R}}$, so that the loop group action becomes more and more nearly unitary. We then want to restrict attention to the loop-invariant subspace and apply the Segal-Bargmann transform. However, if we simply use the ordinary transform $B_{s}$, it will not make sense in the $s \rightarrow \infty$ limit. So we introduce a "two-parameter transform" $B_{s, t}$. This transform is given by the same formula as the time $t$ transform $B_{t}$, but defined on the time $s$ Hilbert space $L^{2}\left(\bar{H}_{\mathbb{R}}, P_{s}\right)$. The target Hilbert space is $\mathcal{H} L^{2}\left(\bar{H}_{\mathbb{R}}, M_{s, t}\right)$, where $M_{s, t}$ is an appropriately defined Gaussian measure on $\bar{H}_{\mathbb{R}}$. (See [DH], [H5] for details.)

Theorem 21. The following diagram commutes, and all maps are unitary.

$$
\begin{array}{ccc}
L^{2}\left(\bar{H}_{\mathbb{R}}, P_{s}\right)^{\mathcal{L}(K)} & \stackrel{B_{s, t}}{\longleftrightarrow} & \mathcal{H} L^{2}\left(\bar{H}_{\mathbb{C}}, M_{s, t}\right)^{\mathcal{L}(K)} \\
\uparrow \circ h & & \uparrow \circ h_{\mathbb{C}} \\
L^{2}\left(\stackrel{K}{K}, \rho_{s}\right) & \stackrel{B_{s, t}}{\longleftrightarrow} & \mathcal{H} L^{2}\left(K_{\mathbb{C}}, \mu_{s, t}\right)
\end{array}
$$

Here the superscript $\mathcal{L}(K)$ denotes functions invariant under the action of the loop group. On the top row, $B_{s, t}$ is the modified Segal-Bargmann transform for the linear space $\bar{H}_{\mathbb{R}}$. On the bottom row, $B_{s, t}$ is the modified Segal-Bargmann transform for $K$, given by

$$
B_{s, t} f=\text { analytic continuation of } e^{t \Delta_{K} / 2} f \text {. }
$$


It makes sense to take the limit $s \rightarrow \infty$ in the bottom row, and the result is the $K$-invariant Segal-Bargmann transform

$$
C_{t}: L^{2}(K, d x) \rightarrow \mathcal{H} L^{2}\left(K_{\mathbb{C}}, \nu_{t}\right),
$$

described in Section 3.

To summarize in words: if one takes the modified Segal-Bargmann transform $B_{s, t}$ for $\bar{H}_{\mathbb{R}}$, restricts to the loop-invariant subspace, and lets $s$ tend to infinity, the result is the $K$-invariant Segal-Bargmann transform for $K$. This is consistent with the results of Wren $[\mathrm{Wr}$, who approached the problem in a different way. The proofs in $\left[\mathrm{DH}\right.$ rely on the Hermite decomposition (chaos expansion) for $L^{2}\left(\bar{H}_{\mathbb{R}}, P_{s}\right)$.

Let me close this section by considering these results from the point of view of geometric quantization. (See also the discussion in [H7, Sect. 8].) The invariant form of Segal-Bargmann transform for $\mathbb{R}^{d}$ can be obtained by geometric quantization (the $\mathbb{R}^{d}$ case of what is described in Section 3.2). If one lets $d \rightarrow \infty$, one gets roughly $\mathcal{H} L^{2}\left(\bar{H}_{\mathbb{C}}, M_{s, t}\right)$, where the extra parameter $s \gg t$ is a necessary regularization. So $\mathcal{H} L^{2}\left(\bar{H}_{\mathbb{C}}, M_{s, t}\right)$ may be thought of as the result of applying geometric quantization to the phase space $H_{\mathbb{C}}$. If one then reduces by $\mathcal{L}(K)$, the result (by Theorem 21) is naturally identifiable with $\mathcal{H} L^{2}\left(K_{\mathbb{C}}, \nu_{t}\right)$. On the other hand, if we first reduce by $\mathcal{L}(K)$ to get the phase space $T^{*}\left(H_{\mathbb{R}} / \mathcal{L}(K)\right)=T^{*}(K)$, and then perform geometric quantization, Section 3.2 tells us that the result is again $\mathcal{H} L^{2}\left(K_{\mathbb{C}}, \nu_{t}\right)$. So we may say that in this instance "quantization commutes with reduction". That is, there is a natural unitary correspondence between the Hilbert space obtained by first doing geometric quantization and then reducing and the Hilbert space obtained the other way around.

The question of how quantization relates to reduction is an old and important one. V. Guillemin and S. Sternberg GStern sparked a recent surge of interest in the problem by considering it in the context of geometric quantization of compact Kähler manifolds. They consider quantization without the half-forms, and they show that under certain regularity assumptions there is a natural one-to-one linear correspondence between the Hilbert space obtained by first quantizing and then reducing and the one obtained by first reducing and then quantizing. However, they do not prove that this correspondence is unitary, and indeed it seems unlikely to be unitary in general. By contrast, in the Yang-Mills example considered here, we have included the half-forms in the quantization, and we have obtained a unitary correspondence.

Open Problem 9. Give general conditions under which quantization of Kähler manifolds, with or without the half-form correction, commutes unitarily with reduction.

I believe that one is more likely to get a unitary correspondence if one includes the half-forms, but even then I do not expect unitarity in general.

I should add that in the setting of compact Kähler manifolds, as considered in GStern, the Hilbert spaces obtained are finite-dimensional. In that setting an important consequence of the Guillemin-Sternberg correspondence (unitary or not) is that the dimension of the space obtained in the "first quantize and then reduce" approach is the same as the dimension of the space obtained in the "first reduce, then quantize" approach. For non-compact Kähler manifolds the Hilbert spaces are typically infinite-dimensional. 


\section{Proof SKETCHES}

5.1. The Segal-Bargmann transform for $K$. I present here an argument for Theorem 6, as given (in slightly greater generality) in [H5]. The method of proving isometricity is essentially that proposed by T. Thiemann in [T1, Sect. 2.3].

Since $\rho_{t}(x)$ is the heat kernel at the identity, we have that for any function $\phi$ on $K$

$$
\int_{K} \phi(x) \rho_{t}(x) d x=\left(e^{t \Delta_{K} / 2} \phi\right)(e) .
$$

Similarly, if $\psi$ is a function on $K_{\mathbb{C}}$, then

$$
\int_{K_{\mathbb{C}}} \psi(g) \mu_{t}(g) d g=\left(e^{t \Delta_{K_{\mathbb{C}}} / 4} \psi\right)(e) .
$$

Thus the isometricity of the Segal-Bargmann transform is equivalent to the statement that for all $f \in L^{2}\left(K, \rho_{t}\right)$ we have

$$
e^{t \Delta_{K} / 2}(\bar{f} f)(e)=e^{t \Delta_{K_{\mathbb{C}}} / 4}\left(\overline{e^{t \Delta_{K} / 2} f} e^{t \Delta_{K} / 2} f\right)(e) .
$$

On the right we have implicitly analytically continued $e^{t \Delta_{K} / 2} f$ to $K_{\mathbb{C}}$.

Let us assume that $f$ itself admits an analytic continuation to $K_{\mathbb{C}}$. (The space of such $f$ 's is dense.) Now, $\Delta_{K}=\sum X_{k}^{2}$, regarded as a left-invariant differential operator on $K_{\mathbb{C}}$, commutes with complex conjugation and with analytic continuation. Thus

$$
\overline{e^{t \Delta_{K} / 2} f}=e^{t \Delta_{K} / 2} \bar{f}
$$

Note that on the left, we are first applying $e^{t \Delta_{K} / 2}$, then analytically continuing, and then taking the complex conjugate. On the right we are first analytically continuing, then taking the complex conjugate, and then applying $e^{t \Delta_{K} / 2}$.

Next consider the operators

$$
\begin{aligned}
& Z_{k}=\frac{1}{2}\left(X_{k}-i J X_{k}\right) \\
& \bar{Z}_{k}=\frac{1}{2}\left(X_{k}+i J X_{k}\right),
\end{aligned}
$$

which reduce in the case $K_{\mathbb{C}}=\mathbb{C}^{d}$ to $\partial / \partial z_{k}$ and $\partial / \partial \bar{z}_{k}$. On the holomorphic function $f$ we have $Z_{k} f=X_{k} f$ and $\bar{Z}_{k} f=0$, and on the anti-holomorphic function $\bar{f}, Z_{k} \bar{f}=0$ and $\bar{Z}_{k} f=X_{k} f$. It follows that

$$
\overline{e^{t \Delta_{K} / 2} f} e^{t \Delta_{K} / 2} f=e^{t \sum Z_{k}^{2} / 2} e^{t \sum \bar{Z}_{k}^{2} / 2}(\bar{f} f) .
$$

So the desired norm equality becomes

$$
e^{t \Delta_{K} / 2}(\bar{f} f)(e)=e^{t \Delta_{K_{\mathbb{C}}} / 4} e^{t \sum Z_{k}^{2} / 2} e^{t \sum \bar{Z}_{k}^{2} / 2}(\bar{f} f)(e) .
$$

Now, the holomorphic vector field $Z_{k}$ automatically commutes with the antiholomorphic vector field $\bar{Z}_{l}$ (or calculate this directly). Thus the second and third exponents on the right of (15.4) may be combined. The exponent that results is

$$
\frac{t}{2} \sum_{k=1}^{\operatorname{dim} \mathfrak{k}}\left(Z_{k}^{2}+\bar{Z}_{k}^{2}\right)=\frac{t}{4} \sum_{k=1}^{\operatorname{dim} \mathfrak{k}}\left(X_{k}^{2}-\left(J X_{k}\right)^{2}\right) .
$$

This is a constant times the Casimir operator for $K_{\mathbb{C}}$, which is bi-invariant and therefore commutes with the left-invariant operator $\Delta_{K_{\mathbb{C}}}$. So in the end all three exponents on the right in (5.4) may be combined. It thus suffices to have the sum 
of the three exponents on the right in (5.4) equal to the exponent on the left. So we need

$$
\frac{t}{2} \sum_{k=1}^{\operatorname{dim} \mathfrak{k}} X_{k}^{2}=\frac{t}{4} \sum_{k=1}^{\operatorname{dim} \mathfrak{k}} X_{k}^{2}+\frac{t}{4} \sum_{k=1}^{\operatorname{dim} \mathfrak{k}}\left(J X_{k}\right)^{2}+\frac{t}{4} \sum_{k=1}^{\operatorname{dim} \mathfrak{k}}\left(X_{k}^{2}-\left(J X_{k}\right)^{2}\right),
$$

which is true!

It is not difficult to make this argument rigorous on a dense subspace of $L^{2}\left(K, \rho_{t}\right)$, thus establishing the isometricity of the Segal-Bargmann transform $B_{t}$. Using Proposition 3 (see also the appendix of [H5]), the surjectivity can be reduced to two cases: $K=\mathbb{R}^{d}$ and $K$ compact. In the $\mathbb{R}^{d}$ case, one shows that the image of $B_{t}$ contains all holomorphic polynomials, which are known [B] Sect. 1b] to be dense in $\mathcal{H} L^{2}\left(\mathbb{C}^{d}, \mu_{t}\right)$. In the case $K$ compact, it is easily seen that the image of $B_{t}$ contains all the matrix entries for finite-dimensional holomorphic representations of $K_{\mathbb{C}}$. Using the Peter-Weyl theorem and the "averaging lemma" [H1, Lem. 11], one can show that these holomorphic matrix entries are dense in $\mathcal{H} L^{2}\left(K_{\mathbb{C}}, \mu_{t}\right)$ and thus that $B_{t}$ is surjective.

5.2. The Taylor map. The argument used here is essentially that of $[\mathrm{Dr}]$ and DG], except that I give it in "exponentiated form". If $F \in \mathcal{H} L^{2}\left(K_{\mathbb{C}}, \mu_{t}\right)$, then by (5.2)

$$
\begin{aligned}
\|F\|_{L^{2}\left(K_{\mathbb{C}}, \mu_{t}\right)}^{2} & =\int_{K_{\mathbb{C}}}|F(g)|^{2} \mu_{t}(g) d g \\
& =\left.e^{t \Delta_{K_{\mathbb{C}}} / 4}(\bar{F}(g) F(g))\right|_{g=e} .
\end{aligned}
$$

But in the notation of the previous subsection we have

$$
\frac{\Delta_{K_{\mathbb{C}}}}{4}=\sum_{k=1}^{\operatorname{dim} \mathfrak{k}} \bar{Z}_{k} Z_{k}
$$

Since $Z_{k}$ annihilates the anti-holomorphic function $\bar{F}(g)$ and $\bar{Z}_{k}$ annihilates the holomorphic function $F(g)$, when applying $\Delta_{K_{\mathbb{C}}}$ to $\bar{F}(g) F(g)$, only the "cross terms" survive. That is,

$$
\frac{\Delta_{K_{\mathbb{C}}}}{4}(\bar{F}(g) F(g))=\sum_{k=1}^{\operatorname{dim} \mathfrak{k}} \bar{Z}_{k} \bar{F}(g) Z_{k} F(g) .
$$

Also, $\bar{Z}_{k} \bar{F}=X_{k} \bar{F}$ and $Z_{k} F=X_{k} F$. Thus

$$
\frac{\Delta_{K_{\mathbb{C}}}}{4}(\bar{F}(g) F(g))=\sum_{k=1}^{\operatorname{dim} \mathfrak{k}} X_{k} \bar{F}(g) X_{k} F(g) .
$$

More generally,

$$
\left(\frac{\Delta_{K_{\mathbb{C}}}}{4}\right)^{n}(\bar{F}(g) F(g))=\sum_{k_{1}, \cdots, k_{n}=1}^{\operatorname{dim} \mathfrak{k}} X_{k_{1}} \cdots X_{k_{n}} \bar{F}(g) X_{k_{1}} \cdots X_{k_{n}} F(g) .
$$

Thus at least formally we have

$$
\left.e^{t \Delta_{K_{\mathbb{C}}} / 4}(\bar{F}(g) F(g))\right|_{g=e}=\sum_{n=0}^{\infty} \frac{t^{n}}{n !} \sum_{k_{1}, \cdots, k_{n}=1}^{\operatorname{dim} \mathfrak{k}} X_{k_{1}} \cdots X_{k_{n}} \bar{F}(e) X_{k_{1}} \cdots X_{k_{n}} F(e) .
$$

This is just the basis-dependent statement of the isometricity of the Taylor map. 
Note that we do not need to have any commutativity for this argument to work, in contrast to the argument for the isometricity of the Segal-Bargmann transform. This observation is the basis of the results of Driver-Gross [DG] (Theorem [1] of Section 2.2).

Of course the above argument is purely formal. In the compact group case it is not hard to justify it on a dense subspace of $\mathcal{H} L^{2}\left(K_{\mathbb{C}}, \mu_{t}\right)$. Then one has to prove that if $K_{\mathbb{C}}$ is simply connected, the Taylor map is onto $J_{t}^{0}$. Suppose then that $\xi$ is an element of $J_{t}^{0}$. Then using a construction of Gross [G2, Sect. 8], it is not too difficult to produce a holomorphic function $F$ whose derivatives at the identity are given by $\xi$. The construction of $F$ is a "Taylor expansion along paths", with the simple-connectedness used to show independence of path. (See [Dr, Sects. 5 and 6].) So to complete the proof, one merely needs to show that the resulting function $F$ is in $\mathcal{H} L^{2}\left(K_{\mathbb{C}}, \mu_{t}\right)$. Certainly this ought to be the case, since the norm of $F$ in $\mathcal{H} L^{2}\left(K_{\mathbb{C}}, \mu_{t}\right)$ ought to be equal to the norm of $\xi$ in $J_{t}^{0}$. However, the proof of isometricity of the Taylor map works only if one knows ahead of time that $F$ is in $\mathcal{H} L^{2}\left(K_{\mathbb{C}}, \mu_{t}\right)$. Instead, then, one estimates the growth of $F$ and gets bounds (Theorem 12) sufficient to show that $F \in \mathcal{H} L^{2}\left(K_{\mathbb{C}}, \mu_{s}\right)$ for all $s<t$. Then using the Taylor isometry at time $s$, one shows that the norm of $F$ at time $s$ is bounded as $s$ increases to $t$. It is then possible to show that the norm of $F$ at time $t$ is finite as well. (See [Dr, Thm. 5.7].)

5.3. The Hermite expansion. Although the Hermite expansion is just the composition of the Segal-Bargmann transform and the Taylor map, it is illuminating to give a direct proof of its isometricity that does not explicitly involve the complex group $K_{\mathbb{C}}$. As in (5.1) in Section 5.1, the norm of a function $f \in L^{2}\left(K, \rho_{t}\right)$ may be computed as

$$
\|f\|_{L^{2}\left(K, \rho_{t}\right)}^{2}=e^{t \Delta_{K} / 2}(\bar{f} f)(e) .
$$

If we formally expand the heat operator $e^{t \Delta_{K} / 2}$ in powers of the Laplacian, then we will have to apply powers of the Laplacian to the product $\bar{f} f$. This will involve applying the product rule repeatedly. The use of the product rule can be organized as follows:

$$
X_{k}(\bar{f} f)(e)=\left.\left(X_{k}+Y_{k}\right)(\bar{f}(x) f(y))\right|_{x=y=e},
$$

where $X_{k}$ means a derivative in the $x$-variable and $Y_{k}$ means the same derivative but in the $y$-variable. Applying this idea repeatedly, we see that

$$
\Delta_{K}(\bar{f} f)(e)=\left.\sum_{k=1}^{\operatorname{dim} \mathfrak{k}}\left(X_{k}+Y_{K}\right)^{2}(\bar{f}(x) f(y))\right|_{x=y=e}
$$

and more generally

$$
\Delta_{K}^{n}(\bar{f} f)(e)=\left.\left(\sum_{k=1}^{\operatorname{dim} \mathfrak{k}}\left(X_{k}+Y_{k}\right)^{2}\right)^{n}(\bar{f}(x) f(y))\right|_{x=y=e} .
$$

Thus formally we may write

$$
e^{t \Delta_{K} / 2}(\bar{f} f)(e)=\left.\exp \left(\frac{t}{2} \sum_{k=1}^{\operatorname{dim} \mathfrak{k}}\left(X_{k}+Y_{k}\right)^{2}\right)(\bar{f}(x) f(y))\right|_{x=y=e} .
$$


Since all the $X_{k}$ 's automatically commute with the $Y_{l}$ 's (since they act on different variables) we have

$$
\sum_{k=1}^{\operatorname{dim} \mathfrak{k}}\left(X_{k}+Y_{k}\right)^{2}=\sum_{k=1}^{\operatorname{dim} \mathfrak{k}} X_{k}^{2}+\sum_{k=1}^{\operatorname{dim} \mathfrak{k}} Y_{k}^{2}+2 \sum_{k=1}^{\operatorname{dim} \mathfrak{k}} X_{k} Y_{k} .
$$

But $\Sigma X_{k}^{2}$ is a bi-invariant operator on $K$, and so it commutes with each $X_{l}$. Thus in fact all three terms on the right side of (5.7) commute. So we may (formally) factor the exponential on the right in (5.6) into a product of three exponentials. If we let the terms with $X_{k}^{2}$ and $Y_{k}^{2}$ act first, then one will act only on $\bar{f}(x)$ and one will act only on $f(y)$. So we get

$$
e^{t \Delta_{K} / 2}(\bar{f} f)(e)=\left.\exp \left(t \sum_{k=1}^{\operatorname{dim} \mathfrak{k}} X_{k} Y_{k}\right)\left(\left[e^{t \Delta_{K} / 2} \bar{f}(x)\right]\left[e^{t \Delta_{K} / 2} f(y)\right]\right)\right|_{x=y=e} .
$$

Now we may expand out the exponential involving $X_{k} Y_{k}$ to give

$$
\begin{aligned}
& \|f\|_{L^{2}\left(K, \rho_{t}\right)}^{2}=e^{t \Delta_{K} / 2}(\bar{f} f)(e) \\
& \quad=\left.\sum_{n=0}^{\infty} \frac{t^{n}}{n !} \sum_{k_{1}, \cdots, k_{n}=1}^{\operatorname{dim} \mathfrak{k}} X_{k_{1}} \cdots X_{k_{n}} e^{t \Delta_{K} / 2} \bar{f}(x) Y_{k_{1}} \cdots Y_{k_{n}} e^{t \Delta_{K} / 2} f(y)\right|_{x=y=e} .
\end{aligned}
$$

Once we evaluate everything at $e$ there is no need to use different letters for the $x$-derivatives and the $y$-derivatives, and we get simply

$$
\|f\|^{2}=\sum_{n=0}^{\infty} \frac{t^{n}}{n !} \sum_{k_{1}, \cdots, k_{n}=1}^{\operatorname{dim} \mathfrak{k}}\left|X_{k_{1}} \cdots X_{k_{n}} e^{t \Delta_{K} / 2} f(e)\right|^{2} .
$$

This is nothing but the statement of the isometricity of the Hermite expansion from Theorem 13

If $K$ is simply connected, then one can argue directly for the surjectivity of the Hermite expansion as in [Hi1], Hi2]. So we let $\xi$ be an element of $J_{t}^{0}$. We want to construct a function $f$ such that $\xi$ encodes all of the derivatives of $e^{t \Delta_{K} / 2} f$ at the identity. Using "Taylor expansion along paths" as in the last subsection, we can produce a function $F$ whose derivatives at the identity are given by $\xi$. (This is where the simple connectedness of $K$ is used.) We think of $F$ now as simply a function on $K$, even though $F$ extends to a holomorphic function on $K_{\mathbb{C}}$. We have to prove that there exists a function $f \in L^{2}\left(K, \rho_{t}\right)$ such that $F=e^{t \Delta_{K} / 2} f$. Hijab argues first that for all $s<t$ there exists $f_{s}$ with $e^{s \Delta_{K} / 2} f_{s}=F$. Then using the isometricity of the Hermite expansion at time $s$, he argues that the norm of $f_{s}$ remains bounded as $s$ increases to $t$, in which case one may show that $f=\lim _{s \uparrow t} f_{s}$ exists with $e^{t \Delta_{K} / 2} f=F$.

\section{REFERENCES}

[AHS] S. Albeverio, B. Hall, and A. Sengupta, The Segal-Bargmann transform for twodimensional Euclidean quantum Yang-Mills, Infinite Dimensional Anal. Quantum Prob. 2 (1999), 27-49.

[ALMMT] A. Ashtekar, J. Lewandowski, D. Marolf, J. Mourão, and T. Thiemann, Coherent state transforms for spaces of connections, J. Funct. Anal. 135 (1996), 519-551. MR 97d:58031

[BSZ] J. Baez, I. Segal, and Z. Zhou, Introduction to Algebraic and Constructive Quantum Field Theory, Princeton Univ. Press, Princeton, NJ, 1992. [MR 93m:81002]

[B] V. Bargmann, On a Hilbert space of analytic functions and an associated integral transform, Part I, Comm. Pure Appl. Math. 14 (1961), 187-214. MR 28:486 
[Bi] P. Biane, Segal-Bargmann transform, functional calculus on matrix spaces and the theory of semi-circular and circular systems, J. Funct. Anal. 144 (1997), 232-286. MR 97k:22011

[De] T. Deck, Hida distributions on compact Lie groups, Infinite Dimensional Anal. Quantum Prob., to appear.

[Dr] B. Driver, On the Kakutani-Itô-Segal-Gross and Segal-Bargmann-Hall isomorphisms, J. Funct. Anal. 133 (1995), 69-128. MR 97j:22020

[DG] B. Driver and L. Gross, Hilbert spaces of holomorphic functions on complex Lie groups, In: New trends in stochastic analysis (K. Elworthy, S. Kusuoka, and I. Shigekawa, Eds.), World Scientific, Singapore, 1997, pp. 76-106. MR 2000h:46029

[DH] B. Driver and B. Hall, Yang-Mills theory and the Segal-Bargmann transform, Commun. Math. Phys. 201 (1999), 249-290. MR 2000c:58064

[E] M. Engliš, Asymptotic behaviour of reproducing kernels of weighted Bergman spaces, Trans. Amer. Math. Soc. 349 (1997), 3717-3735. MR 97k:46033

[FJ] M. Flensted-Jensen, Spherical functions on a real semisimple Lie group. A method of reduction to the complex case, J. Funct. Anal. 30 (1978), 106-146. MR 80f:43022

[F] G. Folland, Harmonic analysis in phase space, Princeton Univ. Press, Princeton, N.J., 1989. MR 92k:22017

[FS] G. Folland and A. Sitaram, The uncertainty principle: a mathematical survey, J. Fourier Anal. Appl. 3 (1997), 207-238. MR 98f:42006

[Go1] M. Gordina, Holomorphic functions and the heat kernel measure on an infinite dimensional complex orthogonal group, Potential Anal., 12 (2000), 325-357.

[Go2] M. Gordina, Heat kernel analysis and Cameron-Martin subgroup for infinite dimensional groups, J. Funct. Anal. 171 (2000), 192-232. CMP 2000:08

[G1] L. Gross, Abstract Wiener spaces, In: Proceedings of the Fifth Berkeley Symposium on Mathematical Statistics and Probablility, Vol. II, Univ. of California Press, 1967. MR 35:3027

[G2] L. Gross, Uniqueness of ground states for Schrödinger operators over loop groups, J. Funct. Anal. 112 (1993), 373-441. MR 94h:58180

[G3] L. Gross, Analysis on loop groups, In: Stochastic analysis and applications in physics (Funchal, 1993), NATO Adv. Sci. Inst. Ser. C Math. Phys. Sci., 449, Kluwer Acad. Publ., Dordrecht, 1994, pp. 99-118. MR 96j:58023

[G4] L. Gross, Harmonic functions on loop groups, In: Séminaire Bourbaki Vol. 1997/98, Astérisque No. 252, (1998), Exp. No. 846, 5, 271-286. MR 2000c:60084

[G5] L. Gross, The homogeneous chaos over compact Lie groups, In: Stochastic processes: A Festschrift in Honour of Gopinath Kallianpur (S. Cambanis et al., Eds.), Springer, New York, 1993, pp. 117-123. MR 97j:22021

[G6] L. Gross, Harmonic analysis for the heat kernel measure on compact homogeneous spaces, In: Stochastic analysis on infinite-dimensional spaces (Baton Rouge, LA, 1994), (H. Kunita and H.-H. Kuo, Eds.), Pitman Res. Notes Math. Ser., 310, Longman Sci. Tech., Harlow, 1994, pp. 99-110. MR 97g:58178

[G7] L. Gross, Some norms on universal enveloping algebras, Canad. J. Math. 50 (1998), 356-377. MR 2000b: 17015

[G8] L. Gross, A local Peter-Weyl theorem, Trans. Amer. Math. Soc. 352 (2000), 413-427. MR 2000c:22007

[G9] L. Gross, Heat kernel analysis on Lie groups, preprint.

[GM] L. Gross and P. Malliavin, Hall's transform and the Segal-Bargmann map, In: Itô's stochastic calculus and probability theory, (M. Fukushima, N. Ikeda, H. Kunita, and S. Watanabe, Eds.), Springer-Verlag, Berlin/New York, 1996, pp. 73-116. MR 98j:22010

[GStenz] V. Guillemin and M. Stenzel, Grauert tubes and the homogeneous Monge-Ampère equation, J. Differential Geom. 34 (1991), 561-570. MR 93e:32018

[GStern] V. Guillemin and S. Sternberg, Geometric quantization and multiplicities of group representations, Invent. Math. 67 (1982), 515-538. MR 83m:58040]

[H1] B. Hall, The Segal-Bargmann "coherent state" transform for compact Lie groups, J. Funct. Anal. 122 (1994), 103-151. MR 95e:22020

[H2] B. Hall, The inverse Segal-Bargmann transform for compact Lie groups, J. Funct. Anal. 143 (1997), 98-116. MR 98e:22004

[H3] B. Hall, Phase space bounds for quantum mechanics on a compact Lie group, Comm. Math. Phys. 184 (1997), 233-250. MR 98g:81108 
[H4] B. Hall, Quantum mechanics in phase space, In: Perspectives on quantization (L. Coburn and M. Rieffel, Eds), Contemp. Math., Vol. 214, Amer. Math. Soc., Providence, RI, 1998, pp. 47-62. MR 99e:22015

[H5] B. Hall, A new form of the Segal-Bargmann transform for Lie groups of compact type, Canad. J. Math. 51 (1999), 816-834. MR 2000g:22013

[H6] B. Hall, Holomorphic methods in analysis and mathematical physics, In: First Summer School in Analysis and Mathematical Physics (S. Pérez-Esteva and C. Villegas-Blas, Eds.), Contemp. Math., Vol. 260, Amer. Math. Soc., Providence, RI, 2000, pp. 1-59.

[H7] B. Hall, Coherent states, Yang-Mills theory, and reduction, preprint. [http://xxx.lanl.gov, quant-ph/9911052]

[H8] B. Hall, Geometric quantization and the generalized Segal-Bargmann transform, in preparation.

[HS] B. Hall and A. Sengupta, The Segal-Bargmann transform for path-groups, J. Funct. Anal. 152 (1998), 220-254. MR 99a:58020

[He] S. Helgason, Differential Geometry, Lie Groups, and Symmetric Spaces, Academic Press, New York, San Diego, 1978. MR 80k:53081

[Hi1] O. Hijab, Hermite functions on compact Lie groups. I, J. Funct. Anal. 125 (1994), 480-492. MR 96e:22017

[Hi2] O. Hijab, Hermite functions on compact Lie groups. II, J. Funct. Anal. 133 (1995), 41-49. MR 96m:22015

[Ho] G. Hochschild, The structure of Lie groups, Holden-Day, San Francisco, 1965. MR 34:7696

[Ki] A. Kirillov, Geometric quantization, In: Dynamical Systems IV (V. Arnold and S. Novikov, Eds.), Encyclopaedia of Mathematical Sciences, Vol. 4, Springer-Verlag, New York, Berlin, 1990.

[KR] K. Kowalski and J. Rembieliński, Coherent states for a particle on a sphere, preprint. [http://xxx.lanl.gov, quant-ph/9912094]

[L1] N. Landsman, Rieffel induction as generalized quantum Marsden-Weinstein reduction, J. Geom. Phys. 15 (1995), 285-319. MR 96k:81116a

[L2] N. Landsman, Mathematical topics between classical and quantum mechanics, SpringerVerlag, New York, 1998. MR 2000g:81081

[LS] L. Lempert and R. Szöke, Global solutions of the homogeneous complex Monge-Ampère equation and complex structures on the tangent bundle of Riemannian manifolds, Math. Ann. 290 (1991), 689-712. MR 92m:32022

[Lo] R. Loll, Non-perturbative solutions for lattice quantum gravity, Nucl. Phys. B 444 (1995), 619-639. MR 96c:83024

[M1] J. Mitchell, Short time behavior of Hermite functions on compact Lie groups, J. Funct. Anal. 164 (1999), 209-248. MR 2000d:22010

[M2] J. Mitchell, Asymptotic expansions of Hermite functions on compact Lie groups, preprint.

[R] J. Rawnsley, A nonunitary pairing of polarizations for the Kepler problem, Trans. Amer. Math. Soc. 250 (1979), 167-180. MR 81m:58037

[RS] M. Reed and B. Simon, Methods of Modern Mathematical Physics, I: Functional Analysis, Second Edition, Academic Press, New York, London, 1980. MR 85e:46002

[Sa] G. Sadasue, Equivalence-singularity dichotomy for the Wiener measures on path groups and loop groups, J. Math. Kyoto Univ. 35 (1995), 653-662. MR 98d:60108

[S1] I. Segal, Mathematical problems of relativistic physics, Chap. VI, In: Proceedings of the Summer Seminar, Boulder, Colorado, 1960, Vol. II. (M. Kac, Ed.), Lectures in Applied Mathematics, American Math. Soc., Providence, Rhode Island, 1963. MR 26:1774

[S2] I. Segal, Mathematical characterization of the physical vacuum for a linear Bose-Einstein field, Illinois J. Math. 6 (1962), 500-523. MR 26:1075

[S3] I. Segal, The complex wave representation of the free Boson field, In: Topics in functional analysis: Essays dedicated to M.G. Krein on the occasion of his 70th birthday (I. Gohberg and M. Kac, Eds), Advances in Mathematics Supplementary Studies, Vol. 3, Academic Press, New York, 1978, pp. 321-343. MR 82d:81069

[St] M. Stenzel, The Segal-Bargmann transform on a symmetric space of compact type, J. Funct. Anal. 165 (1999), 44-58. CMP 99:14

[Su] H. Sugita, Holomorphic Wiener function, In: New trends in stochastic analysis (Charingworth, 1994) (K. D. Elworthy, S. Kusuoka, and I. Shigekawa, Eds.), World Sci. Publishing, River Edge, NJ, 1997, pp. 399-415. MR 2000f:60083 
[T1] T. Thiemann, Reality conditions inducing transforms for quantum gauge field theory and quantum gravity, Classical Quantum Gravity 13 (1996), 1383-1403. MR 97g:83055

[T2] T. Thiemann, Gauge field theory coherent states II. Peakedness properties, preprint. [http://xxx.lanl.gov hep-th/0005237]

[Wo] N. Woodhouse, Geometric Quantization, Second Edition, Oxford Univ. Press, Oxford, New York, 1991. MR 94a:58082

[Wr] K. Wren, Constrained quantisation and $\theta$-angles. II, Nuclear Phys. B 521 (1998), 471-502. MR 99g:58019

[Z] F. Zhu, The heat kernel of the second classical domain and of the symmetric space of a normal real form, Chinese J. Contemp. Math. 13 (1992), 181-200. MR 94h:32054

Department of Mathematics, University of Notre Dame, Notre Dame, Indiana 46556

E-mail address: bhall@nd.edu 